Homology, Homotopy and Applications, vol.11(2), 2009, pp.27-48

\title{
EXTENSIONS OF WEYL AND SCHUR FUNCTORS
}

\author{
MARCIN CHAEUPNIK \\ (communicated by Nicholas J. Kuhn)
}

\begin{abstract}
We study Ext-groups in the category of strict polynomial functors. The main result is a computation of the Ext-groups between twisted Weyl and Schur functors for a large class of Young diagrams.
\end{abstract}

\section{Introduction}

This paper is a third part of my work on homological algebra in the category $\mathcal{P}$ of strict polynomial functors. An ultimate goal of this series of papers is computing Extgroups between simple objects in $\mathcal{P}$. I plan to approach this goal by computing the Ext-groups between Frobenius twisted Weyl and Schur functors first. This problem seems to be easier and I believe that once solved, it will be possible, by using ideas of [C3], to extend the computations to the case of two twisted Schur functors and then to simples.

Hence we focus on the groups $\operatorname{Ext}_{\mathcal{P}}^{*}\left(W_{\mu}^{(i+j)}, S_{\lambda}^{(i)}\right)$ for Young diagrams $\mu, \lambda$ of weights respectively $d$ and $d p^{j}$. In [C1, Th. 6.1], I computed these groups for $j=0$. In the present article we start investigating the case of diagrams of different weights, which is substantially more difficult. The main result (Theorem 4.4) computes the Ext-groups between smaller Weyl and larger Schur functors in the case when $\lambda$ can be obtained by a certain process of enlargement $F_{k}$ from a diagram of weight equal to the weight of $\mu$. The main new ingredient is the use of the Schur-de Rham complex (studied in detail in $[\mathbf{C 2}]$ ) which allows one to pass from larger to smaller diagrams.

The paper is organized as follows. In Section 2, after recalling some results and notations from $[\mathbf{C 1}, \mathbf{C 2}]$, the main theorem is formulated. Section 3 contains technical constructions and lemmas needed for its proof. Theorem 4.4 is proved in Section 4. The paper is concluded by a short discussion of the case of arbitrary diagrams in Section 5 .

\section{Acknowledgements}

I wish to thank Stanisław Betley for many stimulating discussions on functor categories and carefully reading the preliminary version of this article.

The author was partially supported by Białynicki-Birula Subsydium of Foundation of Polish Science and the Polish scientific grants (KBN) 1 P03A 005 26, (MNiSW) N N201 387034.

Received February 4, 2008, revised March 2, 2009, April 3, 2009; published on July 27, 2009.

2000 Mathematics Subject Classification: 18G15, 20G10, 20 G15.

Key words and phrases: Ext-groups, Schur functor.

This article is available at http://intlpress.com/HHA/v11/n2/a3

Copyright (C) 2009, International Press. Permission to copy for private use granted. 


\section{Notation and formulation of the main theorem}

We start by recalling some standard combinatorial notions, mainly in order to fix notation. We shall consider Young diagrams, i.e., finite, weakly decreasing sequences of positive integers pictured, as usual, as sets of boxes arranged into rows of lengths given by the numbers in the sequence. For a Young diagram $\lambda=\left(\lambda_{1}, \ldots, \lambda_{k}\right)$, we call the numbers $\lambda_{i}$ its rows, and the numbers $\tilde{\lambda}_{j}:=\#\left\{i: \lambda_{i} \geqslant j\right\}$ its columns. In fact, the sequence $\left(\widetilde{\lambda}_{1}, \ldots, \widetilde{\lambda}_{\lambda_{1}}\right)$ forms another Young diagram denoted $\tilde{\lambda}$ and called the conjugate diagram. The sum $\sum_{i=1}^{k} \lambda_{i}$ is called the weight of $\lambda$ and is denoted $|\lambda|$. There is a natural linear ordering on the set of Young diagrams called the lexicographical ordering. We say that $\mu$ is lexicographically smaller than $\lambda$ (notation: $\mu<\lambda$ ) if, for the smallest $i$ such that $\mu_{i} \neq \lambda_{i}$, we have $\mu_{i}>\lambda_{i}$ (we explain this strange convention in $[\mathbf{C 1}, \S 2])$.

All said above may be generalized in a straightforward way to the case of skew Young diagrams. A skew Young diagram $\lambda$ of weight $d$ is a pair of Young diagrams $\lambda^{\prime} \subseteq \lambda^{\prime \prime}$ (i.e., $\lambda_{i}^{\prime} \leqslant \lambda_{i}^{\prime \prime}$ for all $i$ ) with $\left|\lambda^{\prime \prime}\right|-\left|\lambda^{\prime}\right|=d$. This should be imagined as the set of boxes of $\lambda^{\prime \prime}$ which do not belong to $\lambda^{\prime}$. Such a pair is often denoted by $\lambda^{\prime \prime} / \lambda^{\prime}$ but we shall prefer, whenever possible, one-letter notation to make formulae simpler. Moreover, for skew diagrams $\lambda=\lambda^{\prime \prime} / \lambda^{\prime}, \mu=\mu^{\prime \prime} / \lambda^{\prime}$ where $\lambda^{\prime}, \lambda^{\prime \prime}, \mu^{\prime \prime}$ are "solid" (i.e., not skew) Young diagrams and $\mu^{\prime \prime} \subseteq \lambda^{\prime \prime}$, we define a skew diagram $\lambda / \mu:=\lambda^{\prime \prime} / \mu^{\prime \prime}$.

Throughout this article we work in the category $\mathcal{P}$ of strict polynomial functors over a fixed field $\mathbf{k}$ of characteristic $p>0$ (cf. [FS, $\S 2]$ ). The simplest examples of strict polynomial functors are $d$-fold tensor, exterior, symmetric and divided power functors denoted respectively by $I^{d}, \Lambda^{d}, S^{d}, D^{d}$. We recall that in a positive characteristic, the last two $S^{d}(V):=\left(V^{\otimes d}\right)_{\Sigma_{d}}$ (coinvariants of the permutation action of the symmetric group) and $D^{d}(V):=\left(V^{\otimes d}\right)^{\Sigma_{d}}$ (invariants of this action) are not isomorphic functors. It is also convenient to label tensor products of such functors by skew Young diagrams. Namely, for $\lambda / \mu=\left(\lambda_{1}, \ldots, \lambda_{k}\right) /\left(\mu_{1}, \ldots, \mu_{k}\right)$, we put $\Lambda^{\lambda / \mu}:=\Lambda^{\lambda_{1}-\mu_{1}} \otimes \cdots \otimes \Lambda^{\lambda_{k}-\mu_{k}}$ and we define $S^{\lambda / \mu}$ and $D^{\lambda / \mu}$ analogously. Moreover, for a skew Young diagram $\lambda$ we define the Schur functor $S_{\lambda}$ to be the image of the composition of natural transformations $\Lambda^{\lambda} \longrightarrow I^{d} \longrightarrow S^{\widetilde{\lambda}}$, where the arrows are respectively the comultiplication and multiplication map (cf. [ABW, §11.1]). We call the epimorphism $\Lambda^{\lambda} \longrightarrow S_{\lambda}$ and monomorphism $S_{\lambda} \longrightarrow S^{\tilde{\lambda}}$ the structural maps. For a strict polynomial functor $F$ we can form its $i$-fold Frobenius twist $F^{(i)}$ defined by the formula $F^{(i)}(V):=F\left(V^{(i)}\right)$, where $V^{(i)}$ stands for the space $V$ with the action of scalars induced by $i$-times iteration of the Frobenius homomorphism, i.e., $x . v:=x^{p^{i}} v$ (see [FS, §1]). We also have a contravariant involution in $\mathcal{P}$ called Kuhn duality. We put $F^{\#}(V):=\left(F\left(V^{*}\right)\right)^{*}$ and it is easy to see that $\left(S^{d}\right)^{\#}=D^{d},\left(\Lambda^{d}\right)^{\#}=\Lambda^{d}$ and more generally $\left(S_{\lambda}\right)^{\#}=W_{\lambda}$. Finally, we recall from, e.g., $\left[\mathbf{C 1}\right.$, p. 779] that $S^{\lambda}$ are injective objects in $\mathcal{P}$ and any $F \in \mathcal{P}$ has a finite injective resolution by sums of $S^{\lambda}$; dually, $D^{\lambda}$ are projective objects in $\mathcal{P}$ and any $F \in \mathcal{P}$ has finite projective resolution by sums of $D^{\lambda}$.

We now recall some technical tools introduced in $[\mathbf{C 1}, \S 2.2]$ which will be used heavily in the present paper. For any skew diagram $\lambda$, the functor in $n$ variables

$$
\left(V_{1}, \ldots, V_{n}\right) \mapsto S_{\lambda}\left(V_{1} \oplus \cdots \oplus V_{n}\right)
$$


has a filtration $M_{\alpha^{1} \subset \ldots \subset \alpha^{n-1} \subset \lambda}$, with ordering coming from the $(n-1)$-fold lexicographic ordering, i.e., to compare sequences $\left(\alpha^{1}, \ldots, \alpha^{n-1}\right)$ and $\left(\alpha^{11}, \ldots, \alpha^{\prime n-1}\right)$ we pick the smallest $i$ such that $\alpha^{i} \neq \alpha^{i}$ and compare lexicographically $\alpha^{i}$ and $\alpha^{i}$. We recall again that $\alpha^{i}$ may be skew; hence, for example, $\alpha^{i} \subseteq \alpha^{i+1}$ means that we have $\alpha^{i}=\alpha^{i^{\prime}} / \beta, \alpha^{i+1}=\alpha^{i+1^{\prime}} / \beta$ for $\alpha^{i^{\prime}}, \alpha^{i+1^{\prime}}, \beta$ solid and $\alpha^{i^{\prime}} \subseteq \alpha^{i+1^{\prime}}$.

The associated graded object of this filtration is

$$
\bigoplus_{\alpha^{1} \subseteq \cdots \subseteq \alpha^{n-1} \subseteq \lambda} S_{\alpha^{1}}\left(V_{1}\right) \otimes S_{\alpha^{2} / \alpha^{1}}\left(V_{2}\right) \otimes \cdots \otimes S_{\lambda / \alpha^{n-1}}\left(V_{n}\right) .
$$

We also have an analogous filtration for Weyl functors and for Frobenius twisted Schur or Weyl functors. We call this filtration the Decomposition Formula.

This filtration yields a spectral sequence called the Decomposition Spectral Sequence. Let $F_{1}, \ldots, F_{n} \in \mathcal{P}$. Then there is a spectral sequence converging to

$$
\operatorname{Ext}_{\mathcal{P}}^{*}\left(F_{1} \otimes \cdots \otimes F_{n}, S_{\lambda}^{(i)}\right),
$$

whose $E_{1}$-term has the form

$$
E_{1}^{s t}=\bigoplus_{s_{1}+\cdots+s_{n}=s+t} \operatorname{Ext}_{\mathcal{P}}^{s_{1}}\left(F_{1}, S_{\alpha^{1}}^{(i)}\right) \otimes \cdots \otimes \operatorname{Ext}_{\mathcal{P}}^{s_{n}}\left(F_{n}, S_{\lambda / \alpha^{n-1}}^{(i)}\right)
$$

where $t$ stands for the place of $\left(\alpha^{1}, \ldots, \alpha^{n-1}\right)$ in the $(n-1)$-fold lexicographic ordering.

Analogous sequences also exist for

$$
\begin{gathered}
\operatorname{Ext}_{\mathcal{P}}^{*}\left(F_{1} \otimes \cdots \otimes F_{n}, W_{\lambda}^{(i)}\right), \\
\operatorname{Ext}_{\mathcal{P}}^{*}\left(S_{\lambda}^{(i)}, F_{1} \otimes \cdots \otimes F_{n}\right),
\end{gathered}
$$

etc.

The Decomposition Formula for $\lambda=\left(1^{d}\right)$ and $\lambda=(d)$ reduces to the well-known formulae:

$$
\begin{aligned}
& S^{d}\left(V_{1} \oplus \cdots \oplus V_{n}\right)=\bigoplus_{s_{1}+\cdots+s_{n}=d} S^{s_{1}}\left(V_{1}\right) \otimes \cdots \otimes S^{s_{n}}\left(V_{n}\right), \\
& D^{d}\left(V_{1} \oplus \cdots \oplus V_{n}\right)=\bigoplus_{s_{1}+\cdots+s_{n}=d} D^{s_{1}}\left(V_{1}\right) \otimes \cdots \otimes D^{s_{n}}\left(V_{n}\right), \\
& \Lambda^{d}\left(V_{1} \oplus \cdots \oplus V_{n}\right)=\bigoplus_{s_{1}+\cdots+s_{n}=d} \Lambda^{s_{1}}\left(V_{1}\right) \otimes \cdots \otimes \Lambda^{s_{n}}\left(V_{n}\right) .
\end{aligned}
$$

In these cases, the Decomposition Spectral Sequence also splits and we get the following isomorphisms for Ext-groups:

$$
\begin{aligned}
& \operatorname{Ext}_{\mathcal{P}}^{*}\left(S^{d}, F_{1} \otimes \cdots \otimes F_{n}\right)=\bigoplus_{s_{1}+\cdots+s_{n}=d} \operatorname{Ext}_{\mathcal{P}}^{*}\left(S^{s_{1}}, F_{1}\right) \otimes \cdots \otimes \operatorname{Ext}_{\mathcal{P}}^{*}\left(S^{s_{n}}, F_{n}\right), \\
& \operatorname{Ext}_{\mathcal{P}}^{*}\left(D^{d}, F_{1} \otimes \cdots \otimes F_{n}\right)=\bigoplus_{s_{1}+\cdots+s_{n}=d} \operatorname{Ext}_{\mathcal{P}}^{*}\left(D^{s_{1}}, F_{1}\right) \otimes \cdots \otimes \operatorname{Ext}_{\mathcal{P}}^{*}\left(D^{s_{n}}, F_{n}\right), \\
& \operatorname{Ext}_{\mathcal{P}}^{*}\left(\Lambda^{d}, F_{1} \otimes \cdots \otimes F_{n}\right)=\bigoplus_{s_{1}+\cdots+s_{n}=d} \operatorname{Ext}_{\mathcal{P}}^{*}\left(\Lambda^{s_{1}}, F_{1}\right) \otimes \cdots \otimes \operatorname{Ext}_{\mathcal{P}}^{*}\left(\Lambda^{s_{n}}, F_{n}\right)
\end{aligned}
$$


In many cases these formulae simplify even more thanks to the decomposition of abelian categories

$$
\mathcal{P} \simeq \bigoplus_{d=0}^{\infty} \mathcal{P}_{d},
$$

where $\mathcal{P}_{d}$ stands for the full subcategory of $\mathcal{P}$ consisting of strict polynomial functors homogeneous of degree $d[\mathbf{F S}, \S 1]$. Thus, since $S^{d}, D^{d}, \Lambda^{d} \in \mathcal{P}_{d}$, we get for $F_{j} \in \mathcal{P}_{s_{j}}$ :

$$
\begin{aligned}
\operatorname{Ext}_{\mathcal{P}}^{*}\left(S^{d}, F_{1} \otimes \cdots \otimes F_{n}\right) & =\operatorname{Ext}_{\mathcal{P}}^{*}\left(S^{s_{1}}, F_{1}\right) \otimes \cdots \otimes \operatorname{Ext}_{\mathcal{P}}^{*}\left(S^{s_{n}}, F_{n}\right), \\
\operatorname{Ext}_{\mathcal{P}}^{*}\left(D^{d}, F_{1} \otimes \cdots \otimes F_{n}\right) & =\operatorname{Ext}_{\mathcal{P}}^{*}\left(D^{s_{1}}, F_{1}\right) \otimes \cdots \otimes \operatorname{Ext}_{\mathcal{P}}^{*}\left(D^{s_{n}}, F_{n}\right), \\
\operatorname{Ext}_{\mathcal{P}}^{*}\left(\Lambda^{d}, F_{1} \otimes \cdots \otimes F_{n}\right) & =\operatorname{Ext}_{\mathcal{P}}^{*}\left(\Lambda^{s_{1}}, F_{1}\right) \otimes \cdots \otimes \operatorname{Ext}_{\mathcal{P}}^{*}\left(\Lambda^{s_{n}}, F_{n}\right) .
\end{aligned}
$$

Similarly to the general case, analogous formulae also hold for $S^{d}, D^{d}, \Lambda^{d}$ as covariant variable and for Frobenius twisted functors. All these special instances of the Decomposition Formula and Decomposition Spectral Sequence will be referred to as the Exponential Formula.

We now recall a calculation achieved in $[\mathbf{C 1}]$. We are interested in the groups $\operatorname{Ext}_{\mathcal{P}}^{*}\left(F^{(i)}, G^{(i)}\right)$ for $F, G \in \mathcal{P}_{d}$ because they are closely related (for large $i$ ) to the groups $\operatorname{Ext}_{G L_{n}(\mathbf{k})}^{*}\left(F\left(\mathbf{k}^{n}\right), G\left(\mathbf{k}^{n}\right)\right)$ (see $\left.[\mathbf{C 1}, \S 1]\right)$. Let $A_{i}$ denote the graded $\mathbf{k}$-space which is one-dimensional in even degrees smaller than $2 p^{i}$ and 0 elsewhere (in fact $A_{i} \simeq \operatorname{Ext}_{\mathcal{P}}^{*}\left(I^{(i)}, I^{(i)}\right)\left[\mathbf{F S}\right.$, Th. 4.5]), and let $B_{i}:=A_{i}^{\otimes d} \otimes \mathbf{k} \Sigma_{d}$, where $\mathbf{k} \Sigma_{d}$ is the kgroup algebra for the symmetric group $\Sigma_{d}$ (in fact $B_{i} \simeq \operatorname{Ext}_{\mathcal{P}}^{*}\left(I^{d(i)}, I^{d(i)}\right)[\mathbf{C 1}, \S 4]$ ). We shall regard $B_{i}$ as a $\mathbf{k} \Sigma_{d}$-bimodule with the left action diagonal and right on the group algebra only (see Section 3 and $[\mathbf{C 1}, \S 4]$ ). Let, at last, $s_{\mu}, s_{\lambda}$ be evident symmetrizations of Schur functors $S_{\mu}, S_{\lambda}$. A symmetrization of a functor $F$ is, roughly speaking, a functor from $\mathbf{k} \Sigma_{d}$-modules to k-modules which should be applied to $V^{\otimes d}$ in order to get $\mathrm{F}(\mathrm{V})$. Thus, for example, $s_{\lambda}$ is a functor which takes a $\mathbf{k} \Sigma_{d^{-}}$-module $M$ to the image of the composition $\left((M)^{\text {alt }}\right)^{\Sigma_{\lambda}} \longrightarrow M \longrightarrow(M)_{\Sigma_{\tilde{\lambda}}}$, where

$$
\Sigma_{\lambda}:=\Sigma_{\lambda_{1}} \times \cdots \times \Sigma_{\lambda_{s}}, M^{\text {alt }}:=M \otimes \operatorname{sgn}
$$

(cf. $[\mathbf{C} 1, \S 3])$. Then

Theorem 2.1 ([C1, Th. 6.1]).

$$
\operatorname{Ext}_{\mathcal{P}}^{*}\left(W_{\mu}^{(i)}, S_{\lambda}^{(i)}\right) \simeq s_{\lambda}\left(s_{\mu}\left(B_{i}\right)\right)
$$

where we apply $s_{\mu}$ to the left $\Sigma_{d}$-structure on $B_{i}$ and then $s_{\lambda}$ to the resulting right $\Sigma_{d}$-module.

Now it is quite natural to try and generalize the above computation to the case of $\operatorname{Ext}_{\mathcal{P}}^{*}\left(W_{\mu}^{(i+j)}, S_{\lambda}^{(i)}\right)$ for $\lambda$ of weight $d p^{j}$. Unfortunately, in general, the pair $W_{\mu}^{(j)}, S_{\lambda}$ does not satisfy the Ext-condition of [C1, Th. 4.4]; hence we should not hope for description of these groups in terms of symmetrizations applied to $B_{i}$ like in $[\mathbf{C 1}$, Thms. 4.4, 6.1].

On the other hand, when we look back at the fundamental computation of $\operatorname{Ext}_{\mathcal{P}}^{*}\left(D^{d(i)}, S^{d(i)}\right)$ [FFSS, Th. 4.5], we see that the authors also consider Ext-groups for diagrams of different weights. In fact, they compute groups $\operatorname{Ext}_{\mathcal{P}}^{*}\left(D^{d(i+j)}, S^{d p^{j}(i)}\right)$ 
by decreasing induction on $j(i+j$ being fixed). The induction step in the proof is achieved by computing hyperExt-groups with coefficients in the de Rham complex $\mathbf{S}^{d p^{j}(i)}$ whose cohomology is a smaller but more twisted de Rham complex $\mathbf{S}^{d p^{j-1}(i+1)}$. This point was my motivation for [C2]: if one wants to compute the Ext-groups for more general Schur functors, then one needs a generalization of the de Rham complex for more general diagrams. Such a generalization, called the Schur-de Rham complex, was introduced and studied in [C2]. Namely, for any skew diagram $\lambda$ of weight $d$ we have a complex $\mathbf{S}_{\lambda}$ of strict polynomial functors homogeneous of degree $d$ (for $\lambda=\left(1^{d}\right)$ we get the de Rham complex $\mathbf{S}^{d}$ ). The cohomology of the Schur-de Rham complex reflects deep combinatorial properties of a diagram. In particular, $\mathbf{S}_{\lambda}$ is acyclic if the $p$-core of $\lambda$ (cf. [JK, Chap. 2.7]) is non-trivial [C2, Fact 4.3], while when the $p$-core is trivial, $H^{*}\left(\mathbf{S}_{\lambda}\right)$ seems to be related to a certain $p$-tuple of diagrams associated with $\lambda$ and called the $p$-quotient of $\lambda$ (cf. [JK, Chap. 2.7]). I have succeeded in making this relationship precise in one important case.

Definition $2.2([\mathbf{C 2}, \S 5])$. Let $F_{k}(\lambda)$ for $0 \leqslant k \leqslant p-1$ denote the diagram with trivial $p$-core and $p$-quotient whose only non-trivial diagram is the $k$ th which is $\lambda$ (for a skew diagram $\lambda=\lambda^{\prime \prime} / \lambda^{\prime}$ we put $\left.F_{k}(\lambda):=F_{k}\left(\lambda^{\prime \prime}\right) / F_{k}\left(\lambda^{\prime}\right)\right)$.

As it was explained in $[\mathbf{C 2}, \S 5], F_{k}(\lambda)$ is a diagram of weight $p|\lambda|$ built out of $\lambda$ in the following manner. We replace boxes in $\lambda$ lying above the principal diagonal with horizontal strips $(p)$, boxes below the diagonal are replaced by vertical strips $\left(1^{p}\right)$ and finally boxes from the diagonal are replaced by segments of shape $\left(k+1,1^{p-k-1}\right)[\mathbf{C 2}, \S 5]$. Thus $F_{k}(\lambda)$ may be thought of as a kind of $p$-times enlargement of $\lambda$, e.g., $\left.F_{0}((d))\right)=(p d), F_{p-1}\left(\left(1^{d}\right)\right)=\left(1^{p d}\right)$, (a number $k$ causes some shifts in cohomology). Now we have

Theorem 2.3 ([C2, Th. 5.3]).

$$
H^{*}\left(\mathbf{S}_{F_{k}(\lambda)}\right) \simeq \mathbf{S}_{\lambda}^{(1)}\left[h_{k}(\lambda)\right]
$$

the shift in grading $h_{k}(\lambda)$ is given by the formula $h_{k}(\lambda)=(p-1) f_{\lambda}+k e_{\lambda}$, where $e_{\lambda}$ is the number of boxes of $\lambda$ lying on the principal diagonal and $f_{\lambda}$ is the number of boxes lying above it.

Observe that this theorem generalizes the Cartier Theorem $H^{*}\left(\mathbf{S}^{d p}\right) \simeq \mathbf{S}^{d(1)}$ used in $[\mathbf{F F S S}]$, since $\mathbf{S}_{\left(1^{d p}\right)}=\mathbf{S}^{(d p)}$ and $F_{0}\left(\left(1^{d}\right)\right)=\left(1^{d p}\right)$.

Now we are ready to formulate our main result.

Theorem 2.4 (Theorem 4.4 below). For any diagrams $\mu, \lambda$ of weight $d$, and any $i, j, k$, we have isomorphisms of graded vector spaces:

$$
\operatorname{Ext}_{\mathcal{P}}^{*}\left(W_{\mu}^{(i+j)}, S_{F_{k}^{j}(\lambda)}^{(i)}\right) \simeq s_{\lambda}\left(s_{\mu}\left(B_{i j}\right)\right)\left[h_{k}^{j}(\lambda)\right] \simeq s_{\mu}\left(s_{\lambda}\left(B_{i j}\right)\right)\left[h_{k}^{j}(\lambda)\right]
$$

The shift is given by the formula $h_{k}^{j}(\lambda)=\left(p^{j}-1\right) f_{\lambda}+k \frac{p^{j}-1}{p-1} e_{\lambda}$, where $e_{\lambda}$ is the number of boxes of $\lambda$ lying on the principal diagonal and $f_{\lambda}$ is the number of boxes lying above it. 
Moreover, for any transformation of symmetrizations $\phi: w_{\mu} \longrightarrow w_{\mu^{\prime}}$, the induced map

$$
\left(\phi^{(i+j)}\right)^{*}: \operatorname{Ext}_{\mathcal{P}}^{*}\left(W_{\mu^{\prime}}^{(i+j)}, S_{F_{k}^{j}(\lambda)}^{(i)}\right) \longrightarrow \operatorname{Ext}_{\mathcal{P}}^{*}\left(W_{\mu}^{(i+j)}, S_{F_{k}^{j}(\lambda)}^{(i)}\right)
$$

is equal to

$$
\phi^{\#}\left(s_{\lambda}\left(B_{i j}\right)\right)\left[h_{k}^{j}(\lambda)\right]: s_{\mu^{\prime}}\left(s_{\lambda}\left(B_{i j}\right)\right)\left[h_{k}^{j}(\lambda)\right] \longrightarrow s_{\mu}\left(s_{\lambda}\left(B_{i j}\right)\right)\left[h_{k}^{j}(\lambda)\right] .
$$

In the above theorem $F_{k}^{j}$ means the $j$-fold iteration of the operation $F_{k}$ and $B_{i j}=$ $A_{i j}^{\otimes d} \otimes \mathbf{k} \Sigma_{d}$ for the graded $\mathbf{k}$-space $A_{i j}$, which is one-dimensional in degrees divisible by $2 p^{j}$ and smaller than $2 p^{i+j}$ and 0 elsewhere (in fact $A_{i j} \simeq \operatorname{Ext}_{\mathcal{P}}^{*}\left(I^{(i+j)}, S^{p^{j}(i)}\right)[\mathbf{F S}$, Th. 4.5], and $B_{i j} \simeq \operatorname{Ext}_{\mathcal{P}}^{*}\left(I^{d(i+j)},\left(S^{p^{j}(i)}\right)^{\otimes d}\right)$ by the Exponential Formula).

Our theorem is a natural generalization of $[\mathbf{C 1}$, Th. 6.1]. Also its proof is a mixture of ideas appearing in the proofs of [FFSS, Th. 4.5], [C1, Th. 4.4], [C1, Th. 6.1]. First we prove the theorem for $\mu=\left(1^{d}\right)$ inductively by using the Schur-de Rham complex and then we get the general case by resolving the first variable by divided powers. The first step is much more difficult. The problem is that we would like to lift some constructions from functors associated to $\lambda$ to those associated to $F_{k}^{j}(\lambda)$ and this may be difficult. For example, lexicographic properties of diagrams do not guarantee us any more the existence of maps $S_{F_{k}^{j}(\lambda)} \longrightarrow S^{F_{k}^{j}(\widetilde{\lambda})}$ or $\Lambda^{F_{k}^{j}(\lambda)} \longrightarrow S_{F_{k}^{j}(\lambda)}$, which could serve as analogies of structural maps $S_{\lambda} \longrightarrow S^{\widetilde{\lambda}}$ or $\Lambda^{\lambda} \longrightarrow S_{\lambda}$. Luckily, we do not need maps between functors but merely between their Ext-groups. But the Schur functors associated to diagrams which form combinatorial obstacles for constructing the analogies of structural maps turn out to have trivial Ext-groups. The situation resembles that in $[\mathbf{C 2}]$ where only after neglecting some acyclic complexes we were able to construct maps on cohomology (in fact, as we will see, the combinatorics in both contexts is similar). In order to make considerations of this sort precise and functorial we shall use a formalism of localization of derived category which will be discussed in the next section.

\section{Acyclic functors and localization}

We start this section by recalling the notion of localization of derived category and pointing out a large class of functors which are trivial in the localized category. Then we construct analogs of structural maps for enlarged diagrams and compute some induced maps of Ext-groups.

\subsection{Localization of derived category and acyclic functors}

Let $\mathbf{D} \mathcal{P}$ denote the derived category of $\mathcal{P}$. This is a triangulated category (i.e., an additive category with a certain family of diagrams called distinguished triangles and a self-equivalence called shift subject to some axioms) and there is an additive functor $i: \mathcal{P} \longrightarrow \mathbf{D} \mathcal{P}$ yielding an isomorphism

$$
\left.\operatorname{Ext}_{\mathcal{P}}^{n}(X, Y)\right) \simeq \operatorname{Hom}_{\mathbf{D} \mathcal{P}}(i(X)[n], i(Y))
$$

for any $X, Y \in \mathcal{P}$, where $i(X)[n]$ stands for the $n$-fold shift of $i(X)$ (for basics on triangulated and derived categories see, e.g., $[\mathbf{G M}])$. 
We need a version of localization at a given object in $\mathcal{P}$, which works in the context of triangulated categories.

Definition 3.1. Given an object $A \in \mathcal{P}$, we say that a triangulated category $\mathbf{D} \mathcal{P}_{A}$ is an $A$-localization of the derived category $\mathbf{D} \mathcal{P}$ if there exists a functor $L: \mathbf{D} \mathcal{P} \longrightarrow$ $\mathbf{D} \mathcal{P}_{A}$ such that:

- $L$ preserves distinguished triangles and shifts.

- For all $X \in \mathcal{P}, n \geqslant 0, L$ induces an isomorphism

$$
\operatorname{Hom}_{\mathbf{D} \mathcal{P}}(i(A)[n], i(X)) \simeq \operatorname{Hom}_{\mathbf{D} \mathcal{P}_{A}}(L(i(A))[n], L(i(X))) .
$$

- If $f: X \longrightarrow Y$ in $\mathcal{P}$ induces an isomorphism

$$
f_{*}: \operatorname{Ext}_{\mathcal{P}}^{n}(A, X) \simeq \operatorname{Ext}_{\mathcal{P}}^{n}(A, Y)
$$

for all $n \geqslant 0$, then $(L \circ i)(f)$ is an isomorphism.

The existence of such a localization is well known (see, e.g., [Ne, Chap. 2.1]. The second condition allows us to carry over calculations of Ext-groups to the localized category; hence from now on we shall not distinguish between $\operatorname{Ext}_{\mathcal{P}}^{n}(A, X)$, $\operatorname{Hom}_{\mathbf{D} \mathcal{P}}(i(A)[n], i(X))$ and $\operatorname{Hom}_{\mathbf{D} \mathcal{P}_{A}}(L(i(A))[n], L(i(X)))$ denoting them all just by $\operatorname{Ext}_{\mathcal{P}}^{n}(A, X)$. The most important is the third condition. Thanks to it, some important maps on Ext-groups which are not induced by any morphisms in $\mathcal{P}$ turn out to be induced by morphisms in $\mathbf{D} \mathcal{P}_{A}$. A typical application is when we have a filtration $\left\{B_{i}\right\}_{i=1}^{k}$ of object $C \in \mathcal{P}$ and $\operatorname{Ext}_{\mathcal{P}}^{n}\left(A, B_{1}\right)=0$ for all $n \geqslant 0$. Then $B_{1}$ is a trivial object in $\mathbf{D} \mathcal{P}_{A}$ (since inclusion $f: 0 \longrightarrow B_{1}$ induces isomorphism on $\operatorname{Ext}_{\mathcal{P}}^{n}(A,-)$ groups). Thus we get a non-trivial morphism $p: B_{2} / B_{1} \longrightarrow C$ in $\mathbf{D} \mathcal{P}_{A}$.

Before formulating our first, rather formal lemma, we recall that $F^{(i)}$ (e.g., $S^{d(i)}$, $\Lambda^{d(i)}$ etc.) always means the $i$-fold Frobenius twist of the functor $F$.

Lemma 3.2. For any diagram $\mu$ of weight $d$, we have the following isomorphisms in the category $\mathbf{D} \mathcal{P}_{W_{\mu}^{(i+j)}}$

$$
S_{F_{k}^{j}(\lambda)}^{(i)} \simeq \Lambda^{d p^{j}(i)}\left[h_{k}^{j}(\lambda)\right]
$$

for an arbitrary diagram $\lambda$ of weight $d$ consisting of a single row, and

$$
S_{F_{k}^{j}\left(\lambda^{\prime}\right)}^{(i)} \simeq S^{d p^{j}(i)}\left[h_{k}^{j}\left(\lambda^{\prime}\right)\right]
$$

for an arbitrary diagram $\lambda^{\prime}$ of weight $d$ consisting of a single column.

The shift is given by the formula $h_{k}^{j}(\rho)=\left(p^{j}-1\right) f_{\rho}+k \frac{p^{j}-1}{p-1} e_{\rho}$, where $e_{\rho}$ is the number of boxes of $\rho$ lying on the principal diagonal and $f_{\rho}$ is the number of boxes of $\rho$ lying above it (we recall that $\rho$ may be a skew diagram; hence its shape does not determine its position with respect to the main diagonal).

Proof. Since the proofs for $\lambda$ and $\lambda^{\prime}$ are analogous, we focus on the case of a one-row diagram $\lambda$. The idea of proof is similar to that of [C2, Lemma 7.1]. We start with recalling some useful constructions from $[\mathbf{C 2}, \S \S 4,7]$. We call a skew Young diagram a skew hook when it does not contain a clock of shape $(2,2)$ and is connected, i.e., we can join every two boxes by a path consisting of boxes where each two consecutive 
boxes have a common edge. Hence the set of boxes of a skew hook can be linearly ordered by saying that the smaller of two boxes is one which is placed higher or more to the right. We say that " $p^{j}$-slices in a skew hook are placed horizontally" when for each $i$ the $\left(i p^{j}+1\right)$ th box with respect to the linear ordering lies to the left of the $\left(i p^{j}\right)$ th box. We observe that $F_{k}^{j}(\lambda)$ for $\lambda$ of weight $d$ consisting of a single row is a skew hook with $p^{j}$-slices placed horizontally.

We shall prove our assertion for a slightly wider class of diagram than it was stated in Lemma 3.2, namely for the class of skew hooks of weight $d p^{j}$ with $p^{j}$-slices placed horizontally. The proof goes by induction on the number of rows in our skew hook $\alpha$. In order to get the assertion for a diagram $\alpha$, we consider the exact sequence

$$
0 \longrightarrow S_{\alpha /\left.\left(\alpha_{1}\right)\right|_{h}\left(\alpha_{1}\right)}^{(i)} \longrightarrow S_{\alpha /\left(\alpha_{1}\right)}^{(i)} \otimes \Lambda^{\alpha_{1}(i)} \longrightarrow S_{\alpha}^{(i)} \longrightarrow 0,
$$

where $\alpha /\left.\left(\alpha_{1}\right)\right|_{h}\left(\alpha_{1}\right)$ is the diagram obtained from $\alpha$ by putting its first row to the right of the second row (see [C2, pp. 715-716]). The existence and exactness of this sequence may be proved by the argument used in [C2, Lemma 7.1]. Since the $p^{j}$-slices of $\alpha$ are placed horizontally, the number $\alpha_{1}$ cannot be divisible by $p^{j}$. Thus $\operatorname{Ext}_{\mathcal{P}}^{*}\left(F^{(i+j)}, \Lambda^{\alpha_{1}(i)}\right)=0$ for any homogeneous $F$ because $\Lambda^{\alpha_{1}(i)} \in \mathcal{P}_{\alpha_{1} p^{i}}$, while $p^{i+j} \mid \operatorname{deg}\left(F^{(i+j)}\right)$. Hence, in the Decomposition Spectral Sequence

$$
\begin{aligned}
E_{1}^{s t} & \left.=\bigoplus_{s_{1}+s_{2}=s+t} \operatorname{Ext}_{\mathcal{P}}^{s_{1}}\left(W_{\beta}^{(i+j)}, S_{\alpha /\left(\alpha_{1}\right)}^{(i)}\right) \otimes \operatorname{Ext}_{\mathcal{P}}^{s_{2}}\left(W_{\mu / \beta}^{(i+j)}, \Lambda^{\alpha_{1}(i)}\right)\right) \\
& \Longrightarrow \operatorname{Ext}_{\mathcal{P}}^{s+t}\left(W_{\mu}^{(i+j)}, S_{\alpha /\left(\alpha_{1}\right)}^{(i)} \otimes \Lambda^{\alpha_{1}(i)}\right)
\end{aligned}
$$

all the rows in $E_{1}$ are trivial. Therefore we conclude that

$$
\operatorname{Ext}_{\mathcal{P}}^{*}\left(W_{\mu}^{(i+j)}, S_{\alpha /\left(\alpha_{1}\right)}^{(i)} \otimes \Lambda^{\alpha_{1}(i)}\right)=0
$$

This means that $S_{\alpha /\left(\alpha_{1}\right)}^{(i)} \otimes \Lambda^{\alpha_{1}(i)}$ is a trivial object in $\mathbf{D} \mathcal{P}_{W_{\mu}^{(i+j)}}$ and we get an isomorphism $S_{\alpha /\left.\left(\alpha_{1}\right)\right|_{h}\left(\alpha_{1}\right)}^{(i)} \simeq S_{\alpha}^{(i)}[-1]$ in the localized derived category. But $\alpha /\left.\left(\alpha_{1}\right)\right|_{h}\left(\alpha_{1}\right)$ is a skew hook with $p^{j}$-slices placed horizontally and has less rows than $\alpha$, so we can apply the induction hypothesis to it. The final formula for shift comes from the fact that $F_{k}^{j}((1))=\left(k \frac{p^{j}-1}{p-1}+1,1^{p^{j}-1-k \frac{p^{j}-1}{p-1}}\right)$.

We now focus on the problem of finding functors which are trivial in the localized category.

Lemma 3.3. Assume that for some $A \in \mathcal{P}, s, i, j$ satisfying $s>i+j$, and for some diagram $\lambda$, we have $\operatorname{Ext}_{\mathcal{P}}^{*}\left(A^{(s)}, S_{F_{k}^{j}(\lambda)}^{(i)}\right) \neq 0$. Then $\lambda$ has a trivial $p$-core.

Proof. The proof falls naturally into three parts.

1. If the assertion holds for $A_{1}$ and $A_{2}$ and all $s>i+j$, then it does so for $A_{1} \otimes A_{2}$. Assume that $\operatorname{Ext}_{\mathcal{P}}^{*}\left(A_{1}^{\left(s_{0}\right)} \otimes A_{2}^{\left(s_{0}\right)}, S_{F_{k}^{j_{0}}(\lambda)}^{\left(i_{0}\right)}\right) \neq 0$. By the Decomposition Spectral Sequence, there must exist $\alpha^{0} \subset F_{k}^{j_{0}}(\lambda)$ such that $\operatorname{Ext}_{\mathcal{P}}^{*}\left(A_{1}^{\left(s_{0}\right)}, S_{\alpha^{0}}^{\left(i_{0}\right)}\right)$ and $\operatorname{Ext}_{\mathcal{P}}^{*}\left(A_{2}^{\left(s_{0}\right)}, S_{F_{k}^{j_{0}}(\lambda) / \alpha^{0}}^{\left(i_{0}\right)}\right)$ are non-trivial. Hence, according to the assertion for $A_{2}$ (and $\left.j=0\right), F_{k}^{j_{0}}(\lambda) / \alpha^{0}$ has a trivial $p$-core. Then, by [C2, Fact 6.1$]$, there 
exists $\alpha^{1} \subset F_{k}^{j_{0}-1}(\lambda)$ such that $\alpha^{0}=F_{k}\left(\alpha^{1}\right)$. Using our assertion again, this time for $j=1$, we conclude that $F_{k}^{j_{0}-1}(\lambda) / \alpha^{1}$ has a trivial $p$-core; hence there exists $\alpha^{2} \subset F_{k}^{j_{0}-2}(\lambda)$ such that $\alpha^{1}=F_{k}\left(\alpha^{2}\right)$ etc. until we get that $\alpha=F_{k}^{j_{0}}\left(\alpha^{j_{0}}\right)$ and $\alpha^{j_{0}}, \lambda / \alpha^{j_{0}}$ have trivial $p$-cores. Therefore, also $\lambda$ has a trivial $p$-core.

2. $A=D^{d(t)}$. We proceed by induction on $d$. Let $d=1$ and assume that the $p$ core of $\lambda$ is non-trivial. Since we are going to consider both the classical Schur complex and the Schur-de Rham complex introduced in $[\mathbf{C 2}]$ we will call the former the Schur-Koszul complex (but we denote both the complexes by $\mathbf{S}_{\lambda}$ ). We start with taking the twisted Schur-de Rham complex $\mathbf{S}_{\lambda}^{(i+j)}$. We recall from [ABW, Cor. V.1.14] that the $l$ th degree component of $\mathbf{S}_{\lambda}^{(i+j)}$ has a filtration with associated graded object $\bigoplus_{|\alpha|=l} W_{\widetilde{\alpha}}^{(i+j)} \otimes S_{\lambda / \alpha}^{(i+j)}$. Since by [C2, Fact 4.3] $\mathbf{S}_{\lambda}^{(i+j)}$ is acyclic and $\operatorname{Ext}_{\mathcal{P}}^{*}\left(I^{(s+t)}, B \otimes C\right)=0$ for all homogeneous functors $B, C$ of positive degree [FS, Th. 2.13], the spectral sequence of this filtration

$$
E_{1}^{k l}=\operatorname{Ext}_{\mathcal{P}}^{k+l}\left(I^{(s+t)},\left(\mathbf{S}_{\lambda}^{(i+j)}\right)^{l}\right) \Longrightarrow \operatorname{hExt}_{\mathcal{P}}^{k+l}\left(I^{(s+t)}, \mathbf{S}_{\lambda}^{(i+j)}\right)
$$

$\left(\operatorname{hExt}{ }_{\mathcal{P}}^{*}\left(I^{(s+t)}, \mathbf{S}_{\lambda}^{(i+j)}\right)\right.$ stands here for the hyperExt-groups of $I^{(s+t)}$ with coefficients in the complex $\mathbf{S}_{\lambda}^{(i+j)}$ (see, e.g., [CE, Chap. XVII]) converges to 0 and may have non-trivial the first or last column only. Hence we obtain

$$
\operatorname{Ext}_{\mathcal{P}}^{*}\left(I^{(s+t)}, S_{\lambda}^{(i+j)}\right) \simeq \operatorname{Ext}_{\mathcal{P}}^{*+|\lambda|-1}\left(I^{(s+t)}, W_{\widetilde{\lambda}}^{(i+j)}\right) .
$$

Applying the same argument to the Schur-Koszul complex, we get a shift in the opposite direction

$$
\operatorname{Ext}_{\mathcal{P}}^{*}\left(I^{(s+t)}, S_{\lambda}^{(i+j)}\right) \simeq \operatorname{Ext}_{\mathcal{P}}^{*-|\lambda|+1}\left(I^{(s+t)}, W_{\widetilde{\lambda}}^{(i+j)}\right)
$$

But by the hypothesis $s>i+j$ we know that $|\lambda|>1$. This means that

$$
\operatorname{Ext}_{\mathcal{P}}^{*}\left(I^{(s+t)}, S_{\lambda}^{(i+j)}\right)=0=\operatorname{Ext}_{\mathcal{P}}^{*}\left(I^{(s+t)}, W_{\widetilde{\lambda}}^{(i+j)}\right) .
$$

Then we shall show in a similar manner that

$$
\operatorname{Ext}_{\mathcal{P}}^{*}\left(I^{(s+t)}, S_{F_{k}(\lambda)}^{(i+j-1)}\right)=0 .
$$

This time the Schur-de Rham complex $\mathbf{S}_{F_{k}(\lambda)}^{(i+j-1)}$ is not acyclic, but since by $[\mathbf{C 2}$, Th. 5.3] the second spectral sequence converging to $\operatorname{hExt}_{\mathcal{P}}^{*}\left(I^{(s+t)}, \mathbf{S}_{F_{k}(\lambda)}^{(i+j-1)}\right)$ is trivial, we still have the shift of grading between $\operatorname{Ext}_{\mathcal{P}}^{*}\left(I^{(s+t)}, S_{F_{k}(\lambda)}^{(i+j-1)}\right)$ and $\operatorname{Ext}_{\mathcal{P}}^{*}\left(I^{(s+t)}, W_{F_{k}(\widetilde{\lambda})}^{(i+j-1)}\right)$, which gives the desired vanishing of the Ext-groups. Repeating this argument we get $\operatorname{Ext}_{\mathcal{P}}^{*}\left(I^{(s+t)}, S_{F_{k}^{q}(\lambda)}^{(i+j-q)}\right)=0$ for larger and larger $q$. At last, for $q=j$, we obtain our assertion.

The proof of the induction step on $d$ is similar. We assume the assertion for all $d<d_{0}$. In order to get it for $d_{0}$ we look at the spectral sequences converging to $\operatorname{hExt}_{\mathcal{P}}^{*}\left(\boldsymbol{\Lambda}^{d_{0}(s+t)}, S_{\lambda}^{(i+j)}\right.$ ) (equipped with the de Rham differential). By the induction hypothesis and Part $\mathbf{1}$, the second spectral sequence is trivial. Since for the same reason the first sequence has at most two non-trivial columns, we 
get the shift of grading. The shift in the opposite direction is provided by the Koszul complex.

3. The general case. For arbitrary $A$ we take a resolution by products of divided powers. By Parts $\mathbf{1}$ and $\mathbf{2}$, the assertion holds for all functors in the resolution. Therefore it also holds for $A$.

Remark. Observe that even for $s=1, i=j=0$, Lemma 3.3 is not obvious. For solid $\lambda$ it follows from the fact that each twisted functor belongs to the trivial block and the Nakayama Conjecture for $\mathcal{P}$ [Do]. But this argument fails for skew diagrams.

We are going to use this lemma to derive a powerful criterion for detecting functors with trivial Ext-groups.

Fact 3.4. If for some $\beta \subseteq F_{k}^{j}(\lambda)$ and some $A \in \mathcal{P}$, $\operatorname{Ext}_{\mathcal{P}}^{*}\left(A^{(i+j)}, S_{F_{k}^{j}(\lambda) / \beta}^{(i)}\right)$ is nontrivial, then $\beta=F_{k}^{j}(\alpha)$ for some $\alpha \subseteq \lambda$.

Proof. Assume that the above Ext-group is non-trivial. Then, by Lemma 3.3 (for $j=0)$, the $p$-core of $\beta$ is trivial. Hence, by [C2, Fact 6.1$]$ we get $\beta=F_{k}\left(\beta^{\prime}\right)$. Iterating this argument we obtain our assertion (we have used a similar trick in the proof of Part 1 of Lemma 3.3).

Observation 3.5. It seems that we had been doing the same work many times in the proofs of Lemma 3.3 and Fact 3.4. Let us look more closely at the underlying combinatorics. Since we are interested in multi-twisted functors, we should (in contrast to [C2]) consider the operation $F_{k}^{j}$ of $j$-fold enlargement of a diagram. It is completely analogous to $F_{k}$, the only difference is that we replace boxes by " $p$-hooks" instead of "p-hooks". Namely, to build $F_{k}^{j}(\lambda)$ out of $\lambda$, we replace boxes in $\lambda$ lying above the diagonal by the horizontal strips of length $p^{j}$, those below it by vertical ones, and we replace boxes lying on the diagonal by segments of shape $F_{k}^{j}((1))=\left(k \frac{p^{j}-1}{p-1}+1,1^{p^{j}-1-k \frac{p^{j}-1}{p-1}}\right)$. Therefore it would be tempting to derive Fact 3.4 from the " $p{ }^{j}$-analogue" of [C2, Fact 6.1] which obviously holds. Unfortunately it is not true that if $\operatorname{Ext}_{\mathcal{P}}^{*}\left(A^{(j)}, S_{\lambda}\right)$ is non-trivial, then $\lambda$ has a trivial $p^{j}$-core (see Fact 5.2).

\subsection{Structural maps for enlarged diagrams and related Ext-groups}

Our next goal is to construct maps $\Lambda^{F_{k}^{j}(\lambda)} \longrightarrow S_{F_{k}^{j}(\lambda)}, S_{F_{k}^{j}(\lambda)} \longrightarrow S^{F_{k}^{j}(\widetilde{\lambda})}$ which could play roles of the structural maps $\Lambda^{\lambda} \longrightarrow S_{\lambda}, S_{\lambda} \longrightarrow S^{\tilde{\lambda}}$. It will turn out that the construction which fails in the category $\mathcal{P}$ is possible in the localized category. In fact, an attempt to construct an arrow $\Lambda_{k}^{F_{k}^{j}(\lambda)} \longrightarrow S_{F_{k}^{j}(\lambda)}$ in the category $\mathcal{P}$ breaks down because the diagram $F_{k}^{j}\left(\left(\lambda_{1}, \ldots, \lambda_{l-1}\right)\right)$ is not the lexicographically smallest subdiagram of a given weight in $F_{k}^{j}\left(\left(\lambda_{1}, \ldots, \lambda_{l}\right)\right)$ etc. But we shall show that all smaller diagrams give trivial objects in $\mathbf{D} \mathcal{P}_{W_{\mu}^{(i+j)}}$. To see this, let us take $\beta \subseteq F_{k}^{j}(\lambda)$ such that $\operatorname{Ext}_{\mathcal{P}}^{*}\left(W_{\mu}^{(i+j)}, S_{\beta}^{(i)} \otimes S_{F_{k}^{j}(\lambda) / \beta}^{(i)}\right) \neq 0$. Then, by the Decomposition Spectral Sequence, there exists $\gamma \subseteq \mu$ of weight $|\beta| / p^{j}$ such that $\operatorname{Ext}_{\mathcal{P}}^{*}\left(W_{\gamma}^{(i+j)}, S_{\beta}^{(i)}\right) \otimes$ $\operatorname{Ext}_{\mathcal{P}}^{*}\left(W_{\mu / \gamma}^{(i+j)}, S_{F_{k}^{j}(\lambda) / \beta}\right)$ is non-trivial. Hence, by Fact $3.4, \beta=F_{k}^{j}(\alpha)$. But among diagrams of the form $F_{k}^{j}\left(\alpha^{\prime}\right)$, our diagram is the smallest (of a given weight). This 
observation (note that the underlying combinatorics is completely analogous to that of the "Homological Decomposition Formula" in $[\mathbf{C 2}, \S 6])$ enables us to construct the arrow

$$
F_{k}^{j(i)}\left(\phi_{\lambda}\right): \Lambda^{F_{k}^{j}(\lambda)(i)} \longrightarrow S_{F_{k}^{j}(\lambda)}^{(i)}
$$

in the category $\mathbf{D} \mathcal{P}_{W_{\mu}^{(i+j)}}$, and by a similar reasoning, the map

$$
F_{k}^{j(i)}\left(\psi_{\lambda}\right): S_{F_{k}^{j}(\lambda)}^{(i)} \longrightarrow S^{F_{k}^{j}(\widetilde{\lambda})(i)}
$$

Next, observe an interesting fact, that the composition $F_{k}^{j(i)}\left(\psi_{\lambda}\right) \circ F_{k}^{j(i)}\left(\phi_{\lambda}\right)$ exists already in $\mathcal{P}$, for it is equal to the composition of the "comultiplication" and "multiplication": $\Lambda^{F_{k}^{j}(\lambda)(i)} \longrightarrow I^{F_{k}^{j}(\lambda)(i)} \longrightarrow S^{F_{k}^{j}(\widetilde{\lambda})(i)}$. In the last formula $I^{F_{k}^{j}(\lambda)(i)}$ stands for the tensor product of twisted Schur functors corresponding to the $p^{j}$-slices in $F_{k}^{j}(\lambda)$ (we recall the interpretation of the operation $F_{k}^{j}$ in terms of $p^{j}$-hooks given in Observation 3.5). The first arrow is the tensor product of $F_{k}^{j(i)}\left(\phi_{\left(\lambda_{s}\right)}\right)$ for all rows of $\lambda$, while the second is the product of $F_{k}^{j(i)}\left(\psi_{\left(\widetilde{\lambda}_{s}\right)}\right)$ for all columns of $\lambda$. It is easy to see (and we have taken advantage of this in $[\mathbf{C 2}, \S 7]$ ) that for diagrams consisting of a single row (or column) the combinatorial obstacles for the existence of maps in $\mathcal{P}$ disappear.

Similarly, in a dual situation we define the "structural maps":

$$
\begin{aligned}
& F_{k}^{j(i)}\left(\phi_{\lambda}^{\#}\right): W_{F_{k}^{j}(\lambda)}^{(i)} \longrightarrow \Lambda_{k}^{F_{k}^{j}(\lambda)(i)}, \\
& F_{k}^{j(i)}\left(\psi_{\lambda}^{\#}\right): D^{F_{k}^{j}(\lambda)(i)} \longrightarrow W_{F_{k}^{j}(\widetilde{\lambda})(i)},
\end{aligned}
$$

whose composition exists in $\mathcal{P}$. The constructions of the structural maps for Schur and Weyl functors have a common generalization to the case of Schur complexes. We recall that the $l$ th degree component of $\mathbf{S}_{F_{k}^{j}(\lambda)}$ has a filtration with associated graded object

$$
\bigoplus_{|\beta|=l} W_{\widetilde{\beta}} \otimes S_{F_{k}^{j}(\lambda) / \beta} .
$$

Hence, applying the above constructions degree-wise, we get the "graded structural maps"

$$
F_{k}^{j(i)}\left(\phi_{\lambda}^{\bullet}\right): \Lambda^{F_{k}^{j}(\lambda)(i)} \longrightarrow \mathbf{S}_{F_{k}^{j}(\lambda)}^{(i)}
$$

and

$$
F_{k}^{j(i)}\left(\psi_{\lambda}^{\bullet}\right): \mathbf{S}_{F_{k}^{j}(\lambda)}^{(i)} \longrightarrow \mathbf{S}^{F_{k}^{j}(\widetilde{\lambda})(i)}
$$

whose composition also exists in $\mathcal{P}$.

Moreover, thanks to Lemma 3.2 and [FFSS, Th. 4.5], we are able to describe the maps induced on Ext-groups by these compositions. A convenient way of writing all these formulae is to express them as results of applications of various symmetrizations to the $\Sigma_{d^{-b i m o d u l e}} B_{i j}$. We recall from Section 2 that $A_{i j} \simeq \operatorname{Ext}_{\mathcal{P}}^{*}\left(I^{(i+j)}, S^{p^{j}(i)}\right)$, 
$B_{i j} \simeq \operatorname{Ext}_{\mathcal{P}}^{*}\left(I^{d(i+j)},\left(S^{p^{j}(i)}\right)^{\otimes d}\right)$, and $B_{i j}=A_{i j}^{\otimes d} \otimes \mathbf{k}\left[\Sigma_{d}\right]$. The graded space $B_{i j}$ is endowed with a structure of $\Sigma_{d}$-bimodule given by the formula known from [C1, $\left.\S 4\right]$

$$
\sigma . a_{1} \otimes \cdots \otimes a_{d} \otimes e_{\tau} \cdot \lambda:=a_{\sigma^{-1}(1)} \otimes \cdots \otimes a_{\sigma^{-1}(d)} \otimes e_{\sigma \tau \lambda} .
$$

We also recall from Section 2 and $[\mathbf{C 1}, \S 4]$ some useful symmetrizations: $s^{\lambda}(M):=$ $(M)_{\Sigma_{\lambda}}, d^{\lambda}(M):=(M)^{\Sigma_{\lambda}}, \lambda_{\text {inv }}^{\lambda}(M):=\left(M^{\text {alt }}\right)^{\Sigma_{\lambda}}$. Then, slightly generalizing the results of [FFSS, §4.5] by means of the Exponential Formula and Lemma 3.2, we obtain

Fact 3.6. The following isomorphisms of graded vector spaces hold:

$$
\begin{aligned}
\operatorname{Ext}_{\mathcal{P}}^{*}\left(D^{d(i+j)}, \Lambda^{F_{k}^{j}(\lambda)(i)}\right) & \simeq \lambda_{i n v}^{\lambda}\left(s^{d}\left(B_{i j}\right)\right)\left[h_{k}^{j}(\lambda)\right]=\Lambda^{\lambda}\left(A_{i j}\right)\left[h_{k}^{j}(\lambda)\right], \\
\operatorname{Ext}_{\mathcal{P}}^{*}\left(D^{d(i+j)}, S^{F_{k}^{j}(\lambda)(i)}\right) & \simeq s^{\lambda}\left(s^{d}\left(B_{i j}\right)\right)\left[h_{k}^{j}(\lambda)\right]=S^{\lambda}\left(A_{i j}\right)\left[h_{k}^{j}(\lambda)\right], \\
\operatorname{Ext}_{\mathcal{P}}^{*}\left(D^{d(i+j)}, D^{F_{k}^{j}(\lambda)(i)}\right) & \simeq d^{\lambda}\left(s^{d}\left(B_{i j}\right)\right)\left[h_{k}^{\prime j}(\lambda)\right]=D^{\lambda}\left(A_{i j}\right)\left[h_{k}^{\prime j}(\lambda)\right], \\
\operatorname{Ext}_{\mathcal{P}}^{*}\left(\Lambda^{d(i+j)}, \Lambda_{k}^{F_{k}^{j}(\lambda)(i)}\right) & \simeq \lambda_{i n v}^{\lambda}\left(\lambda_{i n v}^{d}\left(B_{i j}\right)\right)\left[h_{k}^{j}(\lambda)\right]=D^{\lambda}\left(A_{i j}\right)\left[h_{k}^{j}(\lambda)\right], \\
\operatorname{Ext}_{\mathcal{P}}^{*}\left(\Lambda^{d(i+j)}, S^{F_{k}^{j}(\lambda)(i)}\right) & \simeq s^{\lambda}\left(\lambda_{i n v}^{d}\left(B_{i j}\right)\right)\left[h_{k}^{j}(\lambda)\right]=\Lambda^{\lambda}\left(A_{i j}\right)\left[h_{k}^{j}(\lambda)\right],
\end{aligned}
$$

the shifts are given by the formulae:

$$
\begin{aligned}
h_{k}^{j}(\lambda) & =\left(p^{j}-1\right) f_{\lambda}+k \frac{p^{j}-1}{p-1} e_{\lambda}, \\
h_{k}^{\prime j}(\lambda) & =\left(p^{j}-1\right)\left(2 d-f_{\lambda}\right)-k \frac{p^{j}-1}{p-1} e_{\lambda},
\end{aligned}
$$

where $e_{\lambda}, f_{\lambda}$ denote respectively the number of boxes lying on and above the main diagonal.

Under these identifications, the map induced on $\operatorname{Ext}_{\mathcal{P}}^{*}\left(D^{d(i+j)},-\right)$ by the map $F_{k}^{j(i)}\left(\psi_{\lambda}\right) \circ F_{k}^{j(i)}\left(\phi_{\lambda}\right)$ is equal to $\psi_{\lambda} \circ \phi_{\lambda}\left(s^{d}\left(B_{i j}\right)\right)\left[h_{k}^{j}(\lambda)\right]=\psi_{\lambda} \circ \phi_{\lambda}\left(A_{i j}\right)\left[h_{k}^{j}(\lambda)\right]$, and the one induced by $F_{k}^{j(i)}\left(\phi_{\lambda}^{\#}\right) \circ F_{k}^{j(i)}\left(\psi_{\lambda}^{\#}\right)$ is equal to

$$
\phi_{\lambda}^{\#} \circ \psi_{\lambda}^{\#}\left(s^{d}\left(B_{i j}\right)\right)\left[h_{k}^{\prime j}(\lambda)\right]=\phi_{\lambda}^{\#} \circ \psi_{\lambda}^{\#}\left(A_{i j}\right)\left[h_{k}^{\prime j}(\lambda)\right] .
$$

Similarly, $F_{k}^{j(i)}\left(\psi_{\lambda}\right) \circ F_{k}^{j(i)}\left(\phi_{\lambda}\right)$ induces on $\operatorname{Ext}_{\mathcal{P}}^{*}\left(\Lambda^{d(i+j)},-\right)$ the map

$$
\phi_{\lambda}^{\#} \circ \psi_{\lambda}^{\#}\left(s^{d}\left(B_{i j}\right)\right)\left[h_{k}^{j}(\lambda)\right]=\phi_{\lambda}^{\#} \circ \psi_{\lambda}^{\#}\left(A_{i j}\right)\left[h_{k}^{j}(\lambda)\right] .
$$

By applying these formulae degree-wise and using the Exponential Formula we get formulae for the Ext-groups with coefficients in the respective graded functors. Taking the convention $\operatorname{Ext}_{\mathcal{P}}^{*}\left(F, \mathbf{C}^{\bullet}\right):=\bigoplus_{s} \operatorname{Ext}_{\mathcal{P}}^{*}\left(F, \mathbf{C}^{s}\right)$ (we do not consider any hyperExtgroups here!) we obtain

Fact 3.7. The following isomorphisms of bigraded vector spaces hold:

$$
\begin{aligned}
\left.\operatorname{Ext}_{\mathcal{P}}^{*}\left(D^{d(i+j)}, \boldsymbol{\Lambda}^{F_{k}^{j}(\lambda)(i)}\right)\right) & \simeq \boldsymbol{\lambda}^{\lambda}\left(s^{d}\left(B_{i j}\right)\right)\left[h_{k}^{j}(\lambda)\right]=\boldsymbol{\Lambda}^{\lambda}\left(A_{i j}\right)\left[h_{k}^{\prime j}(\lambda)\right], \\
\operatorname{Ext}_{\mathcal{P}}^{*}\left(D^{d(i+j)}, \mathbf{S}^{F_{k}^{j}(\lambda)(i)}\right) & \simeq \mathbf{s}^{\lambda}\left(s^{d}\left(B_{i j}\right)\right)\left[h_{k}^{j}(\lambda)\right]=\mathbf{S}^{\lambda}\left(A_{i j}\right)\left[h_{k}^{j}(\lambda)\right],
\end{aligned}
$$

where $\boldsymbol{\lambda}^{\lambda}, \mathbf{s}^{\lambda}$ mean the self-evident graded $\Sigma_{d}$-functors. Under these isomorphisms, the map induced on $\operatorname{Ext}_{\mathcal{P}}^{*}\left(D^{d(i+j)},-\right)$ by the composition $F_{k}^{j(i)}\left(\psi_{\lambda}^{\bullet}\right) \circ F_{k}^{j(i)}\left(\phi_{\lambda}^{\bullet}\right)$ is equal to $\psi_{\lambda}^{\bullet} \circ \phi_{\lambda}^{\bullet}\left(A_{i j}\right)$. 


\section{The main theorem}

We outlined in Section 2 the strategy of computing $\operatorname{Ext}_{\mathcal{P}}^{*}\left(W_{\mu}^{(i+j)}, S_{F_{k}^{j}(\lambda)}^{(i)}\right)$. First we compute $\operatorname{Ext}_{\mathcal{P}}^{*}\left(D^{\mu(i+j)}, S_{F_{k}^{j}(\lambda)}^{(i)}\right)$ by manipulating the second variable and using Fact 3.7. Then we take a twisted projective resolution of the first variable and get the general formula. The first step is much more difficult because the operation $F_{k}^{j}$ is involved here. Although we have succeeded in constructing the "structural arrow" $F_{k}^{j}\left(\psi_{\lambda}\right)$, I was not able to conduct the proof along the lines of the proof of $[\mathbf{C 1}$, Th. 4.3]. The problem is that after lifting a resolution to the level of $F_{k}^{j}$ it is difficult to show that we have obtained an exact complex in the sense of the triangulated structure in $\mathbf{D} \mathcal{P}_{D^{\mu(i+j)}}$. Thus I was forced to come back to ideas of [FLS, FS, FFSS], and prove the formula inductively using the (Schur)-de Rham complex. The difference with [FFSS] is that we perform induction by decreasing the number of twists (the starting point is provided by [C1, Th. 6.1]).

Theorem 4.1. For any diagram $\lambda$ of weight $d$, the map

$$
F_{k}^{j(i)}\left(\psi_{\lambda}^{\bullet}\right): \mathbf{S}_{F_{k}^{j}(\lambda)}^{(i)} \longrightarrow \mathbf{S}^{F_{k}^{j}(\widetilde{\lambda})(i)}
$$

induces a monomorphism

$$
\left(F_{k}^{j(i)}\left(\psi_{\lambda}^{\bullet}\right)\right)_{*}: \operatorname{Ext}_{\mathcal{P}}^{*}\left(D^{d(i+j)}, \mathbf{S}_{F_{k}^{j}(\lambda)}^{(i)}\right) \longrightarrow \operatorname{Ext}_{\mathcal{P}}^{*}\left(D^{d(i+j)}, \mathbf{S}_{k}^{F_{k}^{j}(\widetilde{\lambda})(i)}\right),
$$

whose image is $\mathbf{S}_{\lambda}\left(A_{i j}\right)\left[h_{k}^{j}(\lambda)\right.$ ] (we recall that for a complex $\mathbf{C}^{\bullet}$, $\operatorname{Ext}_{\mathcal{P}}^{*}\left(F, \mathbf{C}^{\bullet}\right)$ means just $\left.\bigoplus_{s} \operatorname{Ext}_{\mathcal{P}}^{*}\left(F, \mathbf{C}^{s}\right)\right)$.

The shift is given by the formula $h_{k}^{j}(\lambda)=\left(p^{j}-1\right) f_{\lambda}+k \frac{p^{j}-1}{p-1} e_{\lambda}$, where $e_{\lambda}$ is the number of boxes of $\lambda$ lying on the principal diagonal and $f_{\lambda}$ is the number of boxes lying above it.

Under the above and Lemma 3.7 identifications, the map

$$
\left(F_{k}^{j(i)}\left(\phi_{\lambda}^{\bullet}\right)\right)_{*}: \operatorname{Ext}_{\mathcal{P}}^{*}\left(D^{d(i+j)}, \boldsymbol{\Lambda}_{k}^{F_{k}^{j}(\lambda)(i)}\right) \longrightarrow \operatorname{Ext}_{\mathcal{P}}^{*}\left(D^{d(i+j)}, \mathbf{S}_{F_{k}^{j}(\lambda)}^{(i)}\right),
$$

is equal to

$$
\phi_{\lambda}^{\bullet}\left(A_{i j}\right)\left[h_{k}^{j}(\lambda)\right]: \boldsymbol{\Lambda}^{\lambda}\left(A_{i j}\right)\left[h_{k}^{j}(\lambda)\right] \longrightarrow \mathbf{S}_{\lambda}\left(A_{i j}\right)\left[h_{k}^{j}(\lambda)\right] .
$$

Proof. We prove our theorem for all integers $k$ simultaneously by a double induction: the external on $d$ and internal on $j$. Since the case $d=1$ is trivial, we can turn to the induction step. We shall show our assertion for given $j, d(d>1)$ assuming it for all $d^{\prime}, j^{\prime}$ such that $d^{\prime}<d$, or $d^{\prime}=d, j^{\prime}<j$.

We start with the case $j=0$, which requires a slightly different argument from that for $j>0$. In order to determine the group $\operatorname{Ext}_{\mathcal{P}}^{*}\left(D^{d(i)},\left(\mathbf{S}_{\lambda}^{(i)}\right)^{l}\right)(l$ refers to the grading in the Schur complex) we consider a filtration on $\left(\mathbf{S}_{\lambda}^{(i)}\right)^{l}$ with associated graded object $\bigoplus_{|\alpha|=l} W_{\widetilde{\alpha}}^{(i)} \otimes S_{\lambda / \alpha}^{(i)}$ (cf. [ABW, Cor. V.1.14]). The spectral sequence of this filtration takes the form

$$
E_{1}^{s t}=\bigoplus_{u+v=s+t} \operatorname{Ext}_{\mathcal{P}}^{u}\left(D^{l(i)}, W_{\widetilde{\alpha}}^{(i)}\right) \otimes \operatorname{Ext}_{\mathcal{P}}^{v}\left(D^{d-l(i)}, S_{\lambda / \alpha}^{(i)}\right) \Longrightarrow \operatorname{Ext}_{\mathcal{P}}^{s+t}\left(D^{d(i)},\left(\mathbf{S}_{\lambda}^{(i)}\right)^{l}\right)
$$

where $t$ is the position of $\alpha$ in the lexicographic ordering of subdiagrams in $\lambda$ of 
weight $l$. Thanks to $\left[\mathbf{C 1}\right.$, Cor. 5.3] we know the groups $E_{1}^{* *}$. In particular, they are concentrated in even total degrees (because $\operatorname{Ext}_{\mathcal{P}}^{\text {odd }}\left(D^{d(i)}, F^{(i)}\right)=0$ by $[$ C1, Th. 4.3]). Therefore, the differentials in this spectral sequence are trivial and we obtain

$$
\begin{aligned}
\operatorname{Ext}_{\mathcal{P}}^{*}\left(D^{d(i)},\left(\mathbf{S}_{\lambda}^{(i)}\right)^{l}\right) & \simeq \bigoplus_{|\alpha|=l} \operatorname{Ext}_{\mathcal{P}}^{*}\left(D^{l(i)}, W_{\widetilde{\alpha}}^{(i)}\right) \otimes \operatorname{Ext}_{\mathcal{P}}^{*}\left(D^{d-l(i)}, W_{\lambda / \alpha}^{(i)}\right) \\
& \simeq \bigoplus_{|\alpha|=l}\left(W_{\widetilde{\alpha}} \otimes S_{\lambda / \alpha}\right)\left(A_{i 0}\right)=\mathbf{S}_{\lambda}^{l}\left(A_{i 0}\right) .
\end{aligned}
$$

To get the part of the theorem which concerns the maps $\phi_{\lambda}^{\bullet(i)}$ and $\psi_{\lambda}^{\bullet(i)}$, we observe that it follows from the construction of the filtration that it is compatible with these maps (in fact this filtration is a special case of the filtration giving the Decomposition Formula for Schur complexes [ABW, Cor. V.1.14]). Hence we may apply the induction hypothesis to all factors in the sum for $0<l<d$. But the cases $l=0$ and $l=d$ require some additional argument. In the first case the required description of the maps $\left(\phi_{\lambda}^{(i)}\right)_{*}$ and $\left(\psi_{\lambda}^{(i)}\right)_{*}$ easily follows from $[$ C1, Th. 6.1]. But the case $l=d$ is more difficult. Namely, the fact that $\left(\psi_{\lambda}^{\#(i)}\right)_{*}: \operatorname{Ext}_{\mathcal{P}}^{*}\left(D^{d(i)}, D^{\widetilde{\lambda}(i)}\right) \longrightarrow \operatorname{Ext}_{\mathcal{P}}^{*}\left(D^{d(i)}, W_{\lambda}^{(i)}\right)$ and $\left(\phi_{\lambda}^{\#(i)}\right)_{*}: \operatorname{Ext}_{\mathcal{P}}^{*}\left(D^{d(i)}, W_{\lambda}^{(i)}\right) \longrightarrow \operatorname{Ext}_{\mathcal{P}}^{*}\left(D^{d(i)}, \Lambda^{\lambda(i)}\right)$ are respectively epic and monic follows from $[\mathbf{C 1}$, Lemma 6.4]. But once we know this, the required description follows from the fact that $\left(\phi_{\lambda}^{\#(i)} \circ \psi_{\lambda}^{\#(i)}\right)_{*}: \operatorname{Ext}_{\mathcal{P}}^{*}\left(D^{d(i)}, D^{\widetilde{\lambda}(i)}\right) \longrightarrow \operatorname{Ext}_{\mathcal{P}}^{*}\left(D^{d(i)}, \Lambda^{\lambda(i)}\right)$ may be, by $\left[\mathbf{C 1}\right.$, Th. 6.1], identified with $\phi_{\lambda}^{\#(i)} \circ \psi_{\lambda}^{\#(i)}\left(A_{i 0}\right)$. This completes the proof for $j=0$.

We now focus on the case $j>0$. Let $E_{I}, E_{I I}$ be respectively the first and the second spectral sequence of hyperExt of $D^{d(i+j)}$ with coefficients in the Schur-de Rham complex $\mathbf{S}_{F_{k}^{j}(\lambda)}^{(i)}$. We have

$$
\begin{gathered}
\left(E_{I}\right)_{1}^{s t}=\operatorname{Ext}_{\mathcal{P}}^{s}\left(D^{d(i+j)},\left(\mathbf{S}_{F_{k}^{j}(\lambda)}^{(i)}\right)^{t}\right) \Longrightarrow \operatorname{hExt}_{\mathcal{P}}^{s+t}\left(D^{d(i+j)}, \mathbf{S}_{F_{k}^{j}(\lambda)}^{(i)}\right), \\
\left(E_{I I}\right)_{2}^{s t}=\operatorname{Ext}_{\mathcal{P}}^{s}\left(D^{d(i+j)}, H^{t}\left(\mathbf{S}_{F_{k}^{j}(\lambda)}^{(i)}\right)\right) \Longrightarrow \operatorname{Lxt}_{\mathcal{P}}^{s+t}\left(D^{d(i+j)}, \mathbf{S}_{F_{k}^{j}(\lambda)}^{(i)}\right) .
\end{gathered}
$$

Similarly, let $D$ be the spectral sequence of hyperExt of $D^{d(i+j)}$ with coefficients in the Schur-Koszul complex $\mathbf{S}_{F_{k}^{j}(\lambda)}^{(i)}$. We have

$$
D_{1}^{s t}=\operatorname{Ext}_{\mathcal{P}}^{s}\left(D^{d(i+j)},\left(\mathbf{S}_{F_{k}^{j}(\lambda)}^{(i)}\right)^{t}\right) \Longrightarrow 0 .
$$

We shall compare these spectral sequences with the sequences $E_{I}^{\prime}, E_{I I}^{\prime}, D^{\prime}$ defined analogously for the complex $\mathbf{S}_{k}^{F^{j(i)}(\widetilde{\lambda})}$.

We first examine the sequence $E_{I I}$. Thanks to $[\mathbf{C 2}$, Th. 5.3] and the induction hypothesis for $j^{\prime}=j-1$, we have

$$
\begin{aligned}
\left(E_{I I}\right)_{2}^{s t} & =\operatorname{Ext}_{\mathcal{P}}^{s}\left(D^{d(i+j)}, H^{t}\left(\mathbf{S}_{F_{k}^{j}(\lambda)}^{(i)}\right)^{*}\right) \\
& =\operatorname{Ext}_{\mathcal{P}}^{s}\left(D^{d(i+j)},\left(\mathbf{S}_{F_{k}^{j-1}(\lambda)}^{(i+1)}\right)^{t-h_{k}^{1}\left(F_{k}^{j-1}(\lambda)\right)}\right) \\
& \simeq \mathbf{S}_{\lambda}\left(A_{i+1, j-1}\right),
\end{aligned}
$$

and we know that $F_{k}^{j-1(i+1)}\left(\psi_{\lambda}^{\bullet}\right)$ induces a monomorphism $\left(E_{I I}\right)_{2} \longrightarrow\left(E_{I I}^{\prime}\right)_{2}$, which 
may be identified with the map

$$
\begin{aligned}
\psi_{\lambda}^{\bullet}\left(A_{i+1, j-1}\right)\left[h_{k}^{1}\left(F_{k}^{j-1}(\lambda)\right)\right]: \mathbf{S}_{\lambda}\left(A_{i+1, j-1}\right)\left[h_{k}^{1}\left(F_{k}^{j-1}(\lambda)\right)\right] \\
\longrightarrow \mathbf{S}^{\widetilde{\lambda}}\left(A_{i+1, j-1}\right)\left[h_{k}^{1}\left(F_{k}^{j-1}(\lambda)\right)\right] . i
\end{aligned}
$$

Moreover, as we remember from the proof of [FFSS, Th. 4.5], there is only one nontrivial differential in the sequence $E_{I I}^{\prime}$. Hence $\left(E_{I I}^{\prime}\right)_{2}$ may be viewed as a complex. This complex is the Schur-Koszul complex $\mathbf{S}^{\widetilde{\lambda}}(\delta)\left[h_{k}^{1}\left(F_{k}^{j-1}(\lambda)\right)\right]$ associated to a (shifted) sequence $\mathbf{A}_{i+1, j-1}$ :

$$
0 \longrightarrow A_{i+1, j-1} \stackrel{\delta}{\longrightarrow} A_{i+1, j-1} \longrightarrow 0,
$$

whose differential $\delta$ is a differential in the sequence $E_{I I}$ for $d=1$. It easily follows from the acyclicity of the Schur-Koszul complex associated to the identity map $[\mathbf{A B W}$, V.1.5] that for a general $f: V \longrightarrow W$ we have $H_{*}\left(\mathbf{S}_{\lambda}(f)\right)=\mathbf{S}_{\lambda}\left(f^{\prime}\right)$ as graded functors, where $f^{\prime}: \operatorname{ker}(f) \longrightarrow \operatorname{coker}(f)$ is an arbitrary map (we recall that the vector spaces in a Schur complex depend merely on the source and target of the map). Thus, since it was shown in $\left[\mathbf{F S}\right.$, Th.4.5] that $H_{*}\left(\mathbf{A}_{i+1, j-1}\right)=\mathbf{A}_{i j}$, we obtain

$$
\operatorname{hExt}_{\mathcal{P}}^{*}\left(D^{d(i+j)}, \mathbf{S}_{k}^{j(\widetilde{\lambda})(i)}\right) \simeq \mathbf{S}^{\widetilde{\lambda}}\left(A_{i j}\right)\left[h_{k}^{1}\left(F_{k}^{j-1}(\lambda)\right)\right]
$$

which is yet another translation of the calculations of [FFSS] into a more invariant language. The most important consequence of this point of view is that we still have

$$
\operatorname{hExt}_{\mathcal{P}}^{*}\left(D^{d(i+j)}, \mathbf{S}_{F_{k}^{j}(\lambda)}^{(i)}\right) \simeq \mathbf{S}_{\lambda}\left(A_{i j}\right)\left[h_{k}^{1}\left(F_{k}^{j-1}(\lambda)\right)\right]
$$

and we get an inclusion

$$
f: \operatorname{hExt}_{\mathcal{P}}^{*}\left(D^{d(i+j)}, \mathbf{S}_{F_{k}^{j}(\lambda)}^{(i)}\right) \longrightarrow \operatorname{hExt}_{\mathcal{P}}^{*}\left(D^{d(i+j)}, \mathbf{S}_{k}^{F^{j}(\widetilde{\lambda})(i)}\right) .
$$

We now turn to the spectral sequences $E_{I}, D$. We look at the first pages of these sequences. In the $l$ th column we have the group $\operatorname{Ext}_{\mathcal{P}}^{*}\left(D^{d(i+j)},\left(\mathbf{S}_{F_{k}^{j}(\lambda)}^{(i)}\right)^{l}\right)$. Applying to the second variable the filtration described in [ABW, Cor. V.1.14], we get a spectral sequence converging to $\operatorname{Ext}_{\mathcal{P}}^{*}\left(D^{d(i+j)},\left(\mathbf{S}_{F_{k}^{j}(\lambda)}^{(i)}\right)^{l}\right)$. The first term of this sequence is

$$
E_{1}^{s t}=\bigoplus_{u+v=s+t} \operatorname{Ext}_{\mathcal{P}}^{u}\left(D^{l(i+j)}, W_{F_{k}^{j}(\alpha)}^{(i)}\right) \otimes \operatorname{Ext}_{\mathcal{P}}^{v}\left(D^{d-l(i+j)}, S_{F_{k}^{j}(\lambda / \alpha)}^{(i)}\right),
$$

where $t$ is the position of $\alpha$ in the lexicographic ordering of subdiagrams in $\lambda$ of weight $l / p^{j}$ (the rows with numbers indivisible by $p^{j}$ are trivial by Fact 3.4 ). We note that the groups appearing in this spectral sequence are known by the induction hypothesis for various $d^{\prime}<d$ (we also use here the theorem for $F_{p-1-k}$ ), unless $l=0, d p^{j}$. Our task will be to show that the differentials in this sequence are trivial. The situation is slightly more complicated than that for $j=0$, because this time it does not suffice to observe that $A_{i j}$ is concentrated in even degrees. We should also show that the lowest Ext-degrees of non-trivial elements in all rows have the same parity. To this end, we compute the smallest $u+v$ for which there exists a non-trivial element in

$$
\operatorname{Ext}_{\mathcal{P}}^{u}\left(D^{l(i+j)}, W_{F_{k}^{j}(\alpha)}^{(i)}\right) \otimes \operatorname{Ext}_{\mathcal{P}}^{v}\left(D^{d-l(i+j)}, W_{F_{k}^{j}(\lambda / \alpha)}^{(i)}\right)
$$


This degree, by the induction hypothesis on $d$ (since $l, d-l<d$ ), is equal to

$$
\begin{aligned}
\left(l-f_{\alpha}-e_{\alpha}\right)\left(p^{j}-1\right)+e_{\alpha}\left(2\left(p^{j}-1\right)-\right. & \left.(p-1-k) \frac{p^{j}-1}{p-1}\right) \\
& +\left(f_{\lambda}-f_{\alpha}\right)\left(p^{j}-1\right)+\left(e_{\lambda}-e_{\alpha}\right) k \frac{p^{j}-1}{p-1} .
\end{aligned}
$$

Now we observe that, since the multiplicities of $e_{\alpha}$ and $f_{\alpha}$ are always even, the parity of the whole expression does not depend on $\alpha$. Therefore, we get for all $l \neq 0, d p^{j}$

$$
\begin{aligned}
\operatorname{Ext}_{\mathcal{P}}^{*}\left(D^{d(i+j)},\left(\mathbf{S}_{F_{k}^{j}(\lambda)}^{(i)}\right)^{l}\right) & \simeq \bigoplus_{|\alpha|=l} \operatorname{Ext}_{\mathcal{P}}^{*}\left(D^{l(i+j)}, W_{F_{k}^{j}(\alpha)}^{(i)}\right) \otimes \operatorname{Ext}_{\mathcal{P}}^{*}\left(D^{d-l(i+j)}, W_{F_{k}^{j}(\lambda / \alpha)}^{(i)}\right) \\
& \simeq \bigoplus_{|\alpha|=l}\left(W_{\widetilde{\alpha}} \otimes S_{\lambda / \alpha}\right)\left(A_{i j}\right) \simeq \mathbf{S}_{\lambda}^{l}\left(A_{i j}\right) .
\end{aligned}
$$

In the further part of the proof, shifts of grading will not play an essential role, so we shall skip them in order to simplify notation.

Let

$$
X:=\operatorname{ker}\left(\operatorname{Ext}_{\mathcal{P}}^{*}\left(D^{d(i+j)}, \mathbf{S}_{F_{k}^{j}(\lambda)}^{(i)}\right) \longrightarrow \operatorname{Ext}_{\mathcal{P}}^{*}\left(D^{d(i+j)}, \mathbf{S}_{k}^{F^{j}(\widetilde{\lambda})(i)}\right)\right) .
$$

By the induction hypothesis for $d^{\prime}<d$ and the previous considerations, $X$ is concentrated in the two extreme columns of $E_{I}$ and $D$. We denote by $X_{0}$ its part contained in the 0th column and by $X_{1}$ the part contained in the $\left(d p^{j}\right)$ th column. Let $\partial$ mean the differential in the spectral sequence $D$. Since the sequence $D$ converges to 0 , $\partial^{d p^{j}}\left(X_{1}\right) \subset X_{0}$. Thus we see that $X_{1}$ must be trivial up to the Ext-degree $d p^{j}-2$. We now turn to the sequence $E_{I}$. We denote by $Y$ the subset of the 0th column of $\left(E_{I}\right)_{1}$ consisting of elements surviving to infinity. Since, as we know from [FFSS, Th. 4.5], the differentials in $E_{I}^{\prime}$ are trivial; there are no elements outside $Y$ up to Ext-degree $d p^{j}-2$. It means that there are no differentials in the sequence $E_{I}$ up to the total degree $d p^{j}-2$. Hence, for $s \leqslant d p^{j}-2$, we have

$$
\begin{aligned}
\operatorname{hExt}_{\mathcal{P}}^{s}\left(D^{d(i+j)}, \mathbf{S}_{F_{k}^{j}(\lambda)}^{(i)}\right) & =\bigoplus_{q=0}^{s} \operatorname{Ext}_{\mathcal{P}}^{q}\left(D^{d(i+j)},\left(\mathbf{S}_{F_{k}^{j}(\lambda)}^{(i)}\right)^{s-q}\right) \\
& \simeq\left(\bigoplus_{q=0}^{s-1}\left(\mathbf{S}_{\lambda}^{s-q}\left(A_{i j}\right)\right)^{q}\right) \oplus \operatorname{Ext}_{\mathcal{P}}^{s}\left(D^{d(i+j)}, S_{F_{k}^{j}(\lambda)}^{(i)}\right) .
\end{aligned}
$$

But, on the other hand, as we remember from the analysis of the spectral sequence $E_{I I}$

$$
\operatorname{hExt}_{\mathcal{P}}^{s}\left(D^{d(i+j)}, \mathbf{S}_{F_{k}^{j}(\lambda)}^{(i)}\right) \simeq \bigoplus_{q=0}^{s}\left(\mathbf{S}_{\lambda}^{s-q}\left(A_{i j}\right)\right)^{q}
$$

Hence we get

$$
\operatorname{dim}\left(\operatorname{Ext}_{\mathcal{P}}^{s}\left(D^{d(i+j)}, S_{F_{k}^{j}(\lambda)}^{(i)}\right)\right)=\operatorname{dim}\left(\left(\mathbf{S}_{\lambda}^{0}\left(A_{i j}\right)\right)^{s}=\operatorname{dim}\left(\left(S_{\lambda}\left(A_{i j}\right)\right)^{s}\right) .\right.
$$

But since the last space is a subquotient of the first, we obtain

$$
\operatorname{Ext}_{\mathcal{P}}^{s}\left(D^{d(i+j)}, S_{F_{k}^{j}(\lambda)}^{(i)}\right) \simeq\left(S_{\lambda}\left(A_{i j}\right)\right)^{s} .
$$


It also means that $X_{0}$ is trivial up to degree $d p^{j}-2$. We now come back to the sequence $D$. On account of the last calculation we get that $X_{1}$ is trivial up to degree $2\left(d p^{j}-2\right)$. This, when we turn again to $E_{I}$, enables us to enlarge the range of degrees in which $Y$ is trivial to $2\left(d p^{j}-2\right)$. As result we obtain the required computation of $\operatorname{Ext}_{\mathcal{P}}^{s}\left(D^{d(i+j)}, S_{F_{k}^{j}(\lambda)}^{(i)}\right)$ and the triviality of $X_{0}$ up to degree $2\left(d p^{j}-2\right)$. Iterating this argument (strictly speaking we apply here the third induction, this time on Extdegree) we conclude that $X=0$,

$$
\operatorname{Ext}_{\mathcal{P}}^{*}\left(D^{d(i+j)}, S_{F_{k}^{j}(\lambda)}^{(i)}\right) \simeq S_{\lambda}\left(A_{i j}\right)
$$

and that the differentials in $E_{I}$ are trivial. The last two facts also show, by dimension counting, that

$$
\operatorname{Ext}_{\mathcal{P}}^{*}\left(D^{d(i+j)}, W_{F_{k}^{j}(\lambda)}^{(i)}\right) \simeq W_{\widetilde{\lambda}}\left(A_{i j}\right)
$$

This completes the proof of the description of the groups $\operatorname{Ext}_{\mathcal{P}}^{*}\left(D^{d(i+j)}, \mathbf{S}_{F_{k}^{j}(\lambda)}^{(i)}\right)$. The last part of the theorem (concerning the arrow $F_{k}^{j}\left(\phi_{\lambda}^{\bullet}\right)$ ) easily follows from the facts we have already proved.

Remark 4.2. Thanks to the Exponential Formula one can immediately generalize Theorem 4.1 to the formula

$$
\operatorname{Ext}_{\mathcal{P}}^{*}\left(D^{\mu(i+j)}, S_{F_{k}^{j}(\lambda)}^{(i)}\right) \simeq s_{\lambda}\left(s_{\mu}\left(B_{i j}\right)\right)\left[h_{k}^{j}(\lambda)\right] \simeq s_{\mu}\left(s_{\lambda}\left(B_{i j}\right)\right)\left[h_{k}^{j}(\lambda)\right]
$$

for an arbitrary diagram $\mu$. We remind the reader that the case of Weyl and Schur functors is very special (see the discussion after [C1, Lemma 6.2]), and we have two alternative descriptions of the Ext-groups here (in general, $\Sigma_{d}$-functors applied to left and right $\Sigma_{d}$-structures need not commute (see the example given in the proof of $[\mathbf{C 1}$, Th. 4.3])). We shall use both descriptions: the first in the proof of Theorem 4.4, the second in the proof of Fact 4.3.

Now, we would like to generalize this computation of Ext-groups to the case of an arbitrary Weyl functor in the first variable. Observe however, that the method of the proof of Theorem 4.1 does not work in this case, because it would require a computation of Ext-groups between two Weyl functors which does not fit our scheme. Luckily, this time we can apply the machinery developed in $[\mathbf{C 1}, \S 3]$, since there are no problems with transformations of the first variable.

Therefore, we should start with understanding the functoriality of the computations achieved in Theorem 4.1.

Fact 4.3. For any transformation of symmetrizations $\phi: d^{\mu} \longrightarrow d^{\mu^{\prime}}$ the induced morphism

$$
\phi^{(i+j) *}: \operatorname{Ext}_{\mathcal{P}}^{*}\left(D^{\mu^{\prime}(i+j)}, S_{F_{k}^{j}(\lambda)}^{(i)}\right) \longrightarrow \operatorname{Ext}_{\mathcal{P}}^{*}\left(D^{\mu(i+j)}, S_{F_{k}^{j}(\lambda)}^{(i)}\right)
$$

is equal to $\phi^{\#}\left(s_{\lambda}\left(B_{i j}\right)\right)$. 
Proof. We consider a commutative diagram (strictly speaking coming from the morphisms in the category $\mathbf{D} \mathcal{P}_{\left.D^{\mu(i+j)} \oplus D^{\mu^{\prime}(i+j)}\right)}$

$$
\begin{array}{rrr}
\operatorname{Ext}_{\mathcal{P}}^{*}\left(D^{\mu^{\prime}(i+j)}, S_{F_{k}^{j}(\lambda)}^{(i)}\right) \stackrel{\phi^{(i+j) *}}{\longrightarrow} & \operatorname{Ext}_{\mathcal{P}}^{*}\left(D^{\mu(i+j)}, S_{F_{k}^{j}(\lambda)}^{(i)}\right) \\
F_{k}^{j(i)}\left(\psi_{\lambda}\right)_{*} \downarrow & \downarrow F^{j(i)}\left(\psi_{\lambda}\right)_{*} \\
\operatorname{Ext}_{\mathcal{P}}^{*}\left(D^{\mu^{\prime}(i+j)}, S^{F_{k}^{j}(\widetilde{\lambda})(i)}\right) \stackrel{\phi^{(i+j) *}}{\longrightarrow} & \operatorname{Ext}_{\mathcal{P}}^{*}\left(D^{\mu(i+j)}, S^{F_{k}^{j}(\widetilde{\lambda})(i)}\right) .
\end{array}
$$

Identifying known groups and arrows we get (up to shift) the diagram

$$
\begin{array}{ccc}
s^{\mu^{\prime}}\left(s_{\lambda}\left(B_{i j}\right)\right) & \stackrel{\phi^{(i+j) *}}{\longrightarrow} & s^{\mu}\left(s_{\lambda}\left(B_{i j}\right)\right) \\
s^{\mu^{\prime}}\left(\psi_{\lambda}\left(B_{i j}\right)\right) \downarrow & \downarrow s^{\mu}\left(\psi_{\lambda}\left(B_{i j}\right)\right) \\
s^{\mu^{\prime}}\left(s^{\lambda}\left(B_{i j}\right)\right) & \stackrel{\phi^{\#}\left(s^{\lambda}\left(B_{i j}\right)\right)}{\longrightarrow} s^{\mu}\left(s^{\lambda}\left(B_{i j}\right)\right) .
\end{array}
$$

Of course, replacing the top arrow by $\phi^{\#}\left(s_{\lambda}\left(B_{i j}\right)\right)$ does not affect the commutativity of the diagram. This, thanks to the monomorphicity of the right vertical arrow, gives our assertion.

We have now all the ingredients we need for the proof of our main result.

Theorem 4.4. For any diagrams $\mu, \lambda$ of weight $d$, and any $i, j, k$, we have isomorphisms of graded vector spaces:

$$
\operatorname{Ext}_{\mathcal{P}}^{*}\left(W_{\mu}^{(i+j)}, S_{F_{k}^{j}(\lambda)}^{(i)}\right) \simeq s_{\lambda}\left(s_{\mu}\left(B_{i j}\right)\right)\left[h_{k}^{j}(\lambda)\right] \simeq s_{\mu}\left(s_{\lambda}\left(B_{i j}\right)\right)\left[h_{k}^{j}(\lambda)\right]
$$

The shift is given by the formula $h_{k}^{j}(\lambda)=\left(p^{j}-1\right) f_{\lambda}+k \frac{p^{j}-1}{p-1} e_{\lambda}$, where $e_{\lambda}$ is the number of boxes of $\lambda$ lying on the principal diagonal and $f_{\lambda}$ is the number of boxes lying above it.

Moreover, for any transformation of symmetrizations $\phi: w_{\mu} \longrightarrow w_{\mu^{\prime}}$, the induced map

$$
\left(\phi^{(i+j)}\right)^{*}: \operatorname{Ext}_{\mathcal{P}}^{*}\left(W_{\mu^{\prime}}^{(i+j)}, S_{F_{k}^{j}(\lambda)}^{(i)}\right) \longrightarrow \operatorname{Ext}_{\mathcal{P}}^{*}\left(W_{\mu}^{(i+j)}, S_{F_{k}^{j}(\lambda)}^{(i)}\right)
$$

is equal to

$$
\phi^{\#}\left(s_{\lambda}\left(B_{i j}\right)\right)\left[h_{k}^{j}(\lambda)\right]: s_{\mu^{\prime}}\left(s_{\lambda}\left(B_{i j}\right)\right)\left[h_{k}^{j}(\lambda)\right] \longrightarrow s_{\mu}\left(s_{\lambda}\left(B_{i j}\right)\right)\left[h_{k}^{j}(\lambda)\right] .
$$

Proof. The proof consists of slightly rearranged elements of the proofs of $[\mathbf{C 1}$, Theorems 4.4,6.1].

We take a finite resolution of $W_{\mu}$ by sums of products of divided powers starting with $\psi_{\mu}^{\#}$. Then we twist it $i+j$ times and apply the functor $\operatorname{Ext}_{\mathcal{P}}^{*}\left(-, S_{F_{k}^{j}(\lambda)}^{(i)}\right)$. In the resulting complex

$0 \rightarrow \operatorname{Ext}_{\mathcal{P}}^{*}\left(W_{\mu}^{(i+j)}, S_{F_{k}^{j}(\lambda)}\right) \stackrel{\psi_{\mu}^{\# *}}{\longrightarrow} \operatorname{Ext}_{\mathcal{P}}^{*}\left(D^{\widetilde{\mu}(i+j)}, S_{F_{k}^{j}(\lambda)} \stackrel{\phi_{1}^{*}}{\longrightarrow} \operatorname{Ext}_{\mathcal{P}}^{*}\left(D^{\mu^{1}(i+j)}, S_{F_{k}^{j}(\lambda)}\right) \rightarrow\right.$ all the groups and arrows starting with the second spot are known by Theorem 4.1 
and Fact 4.3. Hence we have the commutative diagram (in which we omit shifts)

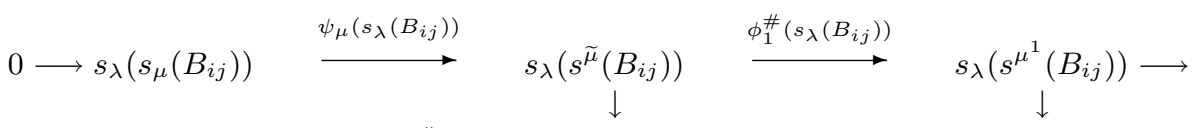

$$
\begin{aligned}
& 0 \longrightarrow \operatorname{Ext}_{\mathcal{P}}^{*}\left(W_{\mu}^{(i+j)}, S_{F_{k}^{j}(\lambda)}\right) \stackrel{\psi_{\mu}^{\# *}}{\longrightarrow} \operatorname{Ext}_{\mathcal{P}}^{*}\left(D^{\widetilde{\mu}(i+j)}, S_{F_{k}^{j}(\lambda)}\right) \stackrel{\phi_{1}^{*}}{\longrightarrow} \operatorname{Ext}_{\mathcal{P}}^{*}\left(D^{\mu^{1}(i+j)}, S_{F_{k}^{j}(\lambda)}\right) \longrightarrow
\end{aligned}
$$

where the vertical arrows are isomorphisms. We now observe that by arguments used in the proof of $[\mathbf{C 1}$, Th. 4.4], the top row is exact (because it is isomorphic to the sequence $(*)$ in $[\mathbf{C 1}$, p. 786$]$ for $\left.F=W_{\mu}, G=S_{\lambda}, f=w_{\mu}, g=s_{\lambda}\right)$. This means that the bottom row is exact starting from the third term. Hence, by standard hyperExtargument we get the exactness of the whole bottom row (see [C1, top of p. 785]). Thus our diagram can be completed by an isomorphism

$$
f: s_{\lambda}\left(s_{\mu}\left(B_{i j}\right)\right) \longrightarrow \operatorname{Ext}_{\mathcal{P}}^{*}\left(W_{\mu}^{(i+j)}, S_{F_{k}^{j}(\lambda)}\right)
$$

We get the assertion concerning functoriality in a similar fashion to that of $[\mathbf{C 1}$, Th. 4.4]; we then finish the proof by the arguments from the proof of [C1, Th. 6.1].

Remark 4.5. We said nothing about the functoriality with respect to the second variable in Theorem 4.4. The reason is that one cannot expect good properties of any morphism $S_{F_{k}^{j}(\lambda)} \longrightarrow S_{F_{k}^{j}\left(\lambda^{\prime}\right)}$. It seems reasonable to consider only maps somehow "induced" by maps $S_{\lambda} \longrightarrow S_{\lambda^{\prime}}$, but then we encounter a problem caused by the fact that the shape of a slice which determines the shift in Ext-grading depends on the position of a box with respect to the main diagonal. We shall illustrate this point by a simple example. For $p=2$ we have a non-trivial transformation $\rho: S^{2} \longrightarrow \Lambda^{2}$. Thus we would expect that the induced map

$$
\rho_{*}: \operatorname{Ext}_{\mathcal{P}}^{*}\left(D^{2(i+1)}, S_{F_{0}\left(\left(1^{2}\right)\right)}^{(i)}\right) \longrightarrow \operatorname{Ext}_{\mathcal{P}}^{*}\left(D^{2(i+1)}, S_{F_{0}((2))}^{(i)}\right)
$$

is equal to $\rho\left(A_{i 1}\right)$. But this cannot happen because $h_{0}^{1}\left(\left(1^{2}\right)\right) \neq h_{0}^{1}((2))$.

\section{Toward the general case}

We now turn to the general situation. Our ultimate goal is to compute the groups $\operatorname{Ext}_{\mathcal{P}}^{*}\left(W_{\mu}^{(i+j)}, S_{\lambda}^{(i)}\right)$ for arbitrary diagrams $\mu, \lambda$ such that $|\mu|=d,|\lambda|=d p^{j}$. By the methods of Section 4, it should be easy to reduce the problem to computing $\operatorname{Ext}_{\mathcal{P}}^{*}\left(D^{d(i+j)}, S_{\lambda}^{(i)}\right)$, and the latter groups should be equal to $\left(\operatorname{Ext}_{\mathcal{P}}^{*}\left(I^{d(i+j)}, S_{\lambda}^{(i)}\right)\right)_{\Sigma_{d}}$. Therefore, if one wants, at least, to understand terms in which the answer should be given, then the most important thing is to describe a structure of $\Sigma_{d}$-module on $\operatorname{Ext}_{\mathcal{P}}^{*}\left(I^{d(i+j)}, S_{\lambda}^{(i)}\right)$ coming from permuting factors in the tensor product. Let us look more closely at the first non-trivial case $i=0, j=1$. Thus we focus on the groups $\operatorname{Ext}_{\mathcal{P}}^{*}\left(I^{d(1)}, S_{\lambda}\right)$ for a diagram $\lambda$ of weight $d p$. By the earlier considerations, this group is trivial if $\lambda$ has a non-trivial $p$-core, and if $\lambda=F_{k}\left(\lambda^{\prime}\right)$ then, according to Theorem 4.4,

$$
\operatorname{Ext}_{\mathcal{P}}^{*}\left(I^{d(1)}, S_{\lambda}\right) \simeq s_{\lambda^{\prime}}\left(B_{01}\right)\left[h_{k}^{1}\left(\lambda^{\prime}\right)\right]=s_{\lambda^{\prime}}\left(\mathbf{k} \Sigma_{d}\right)\left[h_{k}^{1}\left(\lambda^{\prime}\right)\right]=\operatorname{Sp}_{\lambda^{\prime}}\left[h_{k}^{1}\left(\lambda^{\prime}\right)\right]
$$


(we recall that $\mathrm{Sp}_{\lambda}$ means the Specht module associated to the diagram $\lambda$, cf. $[\mathbf{C 1}, \S 3]$, [JK, Chap. 7.1]). In order to understand the situation of a diagram with a trivial $p$-core but having $p$-quotient consisting of several diagrams we shall develop notation introduced in $[\mathbf{C 2}, \S 4]$. We say that $R=\left\{\lambda=\alpha^{0} \supset \alpha^{1} \supset \cdots \supset \alpha^{d}=\emptyset\right\}$ is a decomposition of $\lambda$ into slices if for every $1 \leqslant s \leqslant d$, the diagram $\alpha^{s}$ is obtained from $\alpha^{s-1}$ by removing a rim $p$-hook. In such a situation we call skew hooks $\chi^{s}=\alpha^{s-1} \backslash \alpha^{s}$ the slices of this decomposition. We recall from $[\mathbf{C 2}, \S 4]$ that it may happen that different decompositions produce the same set of slices (in fact this is always the case if $\left.\lambda=F_{k}\left(\lambda^{\prime}\right)\right)$. Let $\left\{R_{t}\right\}_{t=1}^{l}$ be the family of decompositions of $\lambda$ into slices ordered lexicographically and let $\left(\chi_{t}^{1}, \ldots, \chi_{t}^{d}\right)$ be the sequence of slices for $R_{t}$. Then in the Decomposition Spectral Sequence converging to $\operatorname{Ext}_{\mathcal{P}}^{*}\left(I^{d(1)}, S_{\lambda}\right)$ we have

$$
E_{1}^{* t}=\operatorname{Ext}_{\mathcal{P}}^{*}\left(I^{(1)}, S_{\chi_{t}^{1}}\right) \otimes \cdots \otimes \operatorname{Ext}_{\mathcal{P}}^{*}\left(I^{(1)}, S_{\chi_{t}^{d}}\right) \simeq \mathbf{k}\left[h\left(R_{t}\right)-t\right],
$$

where the shift $h\left(R_{t}\right)$ is given by the formula $h\left(R_{t}\right)=\sum_{i} h\left(\chi_{t}^{i}\right)$, and $h\left(\chi_{t}^{i}\right)$ is equal to the number of columns in $\chi_{t}^{i}$ minus 1 . We shall show that the differentials in this spectral sequence are trivial. To do this, it suffices to show that all the numbers $h\left(R_{t}\right)$ have the same parity. But the last statement follows from the fact that the number $(-1)^{h\left(R_{t}\right)}$ is equal to "the sign of the permutation taking the natural ordering of beads before moving them to the configuration corresponding to the core and after it" [JK, p. 81]. For the reader who does not like beads and runners we can give a less elementary argument. Namely, it follows from the Muranghan-Nakayama formula $\left[\mathbf{J K}\right.$, p. 60] that the value of the character of $\mathrm{Sp}_{\lambda}$ on a permutation being a sum of $d$ cycles of length $p$ equals $\sum_{t}(-1)^{h\left(R_{t}\right)}$. On the other hand this value is computed in [JK, p. 83] as $\pm f(\lambda)$ where $f(\lambda)$ is the number of decompositions of $\lambda$ into slices. Therefore all the numbers $(-1)^{h(R)}$ must be equal (of course, beads are hidden in the proof of the formula in [JK, p. 83]). Thus we have shown that

$$
\operatorname{dim}\left(\operatorname{Ext}_{\mathcal{P}}^{*}\left(I^{d(1)}, S_{\lambda}\right)\right)=f(\lambda) .
$$

Moreover, it follows from [JK, Th. 7.27] that for $\lambda$ with a trivial $p$-core and $p$-quotient $\left(q^{0}(\lambda), \ldots, q^{p-1}(\lambda)\right)$ we have

$$
f(\lambda)=\operatorname{dim}\left(\left(\operatorname{Sp}_{q^{0}(\lambda)} \otimes \cdots \otimes \operatorname{Sp}_{q^{p-1}(\lambda)}\right) \uparrow \Sigma_{d}\right),
$$

where $\uparrow \Sigma_{d}$ means the induced $\mathbf{k} \Sigma_{d}$-module. This formula is intriguing, because it suggests the structure of $\Sigma_{d}$-module on $\operatorname{Ext}_{\mathcal{P}}^{*}\left(I^{d(1)}, S_{\lambda}\right)$, which we need to understand. Unfortunately, it is impossible that there is an isomorphism of $\Sigma_{d}$-modules

$$
\operatorname{Ext}_{\mathcal{P}}^{*}\left(I^{d(1)}, S_{\lambda}\right) \simeq\left(\operatorname{Sp}_{q^{0}(\lambda)} \otimes \cdots \otimes \operatorname{Sp}_{q^{p-1}(\lambda)}\right) \uparrow \Sigma_{d}
$$

because the left-hand side is a sum of its homogeneous components, while there is no such decomposition of the right-hand side. But some numerical experiments suggest that, at least in some special cases, the situation might even be simpler.

Conjecture 5.1. There is an isomorphism of $\Sigma_{d}$-modules

$$
\operatorname{Ext}_{\mathcal{P}}^{*}\left(I^{d(1)}, S_{\lambda}\right) \simeq \bigoplus_{\alpha} N_{\alpha ; q^{0}(\lambda), \ldots, q^{p-1}(\lambda)} \operatorname{Sp}_{\alpha}
$$

where $N_{\alpha ; q^{0}(\lambda), \ldots, q^{p-1}(\lambda)}$ is the multiplicity of $\mathrm{Sp}_{\alpha}$ in the Littlewood-Richardson decomposition of $\left(\mathrm{Sp}_{q^{0}(\lambda)} \otimes \cdots \otimes \mathrm{Sp}_{q^{p-1}(\lambda)}\right) \uparrow \Sigma_{d}$. 
We finish this discussion by making one remark concerning the case $j>1$. The strong interplay between $j$-fold Frobenius twist of the first variable and the enlargement $F_{k}^{j}$ of the second variable which we have observed many times (see Observation $3.5)$ could suggest that the group $\operatorname{Ext}_{\mathcal{P}}^{*}\left(D^{d(1)}, S_{\lambda}\right)$ is non-trivial only for $\lambda$ of the form $F_{k}^{j}\left(\lambda^{\prime}\right)$ (this is true for $j=1$ ). But the simplest possible example shows that this is not the case.

Fact 5.2. For $p=2$,

$$
\operatorname{dim}\left(\operatorname{Ext}_{\mathcal{P}}^{n}\left(I^{(2)}, S_{(2,2)}\right)\right)= \begin{cases}1 & n=1,2 \\ 0 & \text { otherwise }\end{cases}
$$

Proof. By the Littlewood-Richardson Rule [Bo] we have a filtration of $S_{(2,1)} \otimes S_{(1)}$ with associated graded object $S_{(3,1)} \oplus S_{(2,2)} \oplus S_{\left(2,1^{2}\right)}$. Hence in the spectral sequence of this filtration for $\operatorname{Ext}_{\mathcal{P}}^{*}\left(I^{(2)},-\right)$, which converges to $\operatorname{Ext}_{\mathcal{P}}^{*}\left(I^{(2)}, S_{(2,1)} \otimes S_{(1)}\right)=0$ we have three non-trivial rows in $E_{1}$ :

$$
\begin{aligned}
& E_{1}^{* 0}=\operatorname{Ext}_{\mathcal{P}}^{*}\left(I^{(2)}, S_{(3,1)}\right)=\operatorname{Ext}_{\mathcal{P}}^{2}\left(I^{(2)}, S_{(3,1)}\right) \simeq \mathbf{k}[2], \\
& E_{1}^{* 1}=\operatorname{Ext}_{\mathcal{P}}^{*+1}\left(I^{(2)}, S_{(2,2)}\right), \\
& E_{1}^{* 2}=\operatorname{Ext}_{\mathcal{P}}^{*+2}\left(I^{(2)}, S_{\left(2,1^{2}\right)}\right)=\operatorname{Ext}_{\mathcal{P}}^{1}\left(I^{(2)}, S_{\left(2,1^{2}\right)}\right) \simeq \mathbf{k}[-1] .
\end{aligned}
$$

Thus we see, that there are two possibilities: either the groups we are interested in have the description predicted by our assertion, or they are trivial. To rule out the second possibility, we consider the spectral sequences converging to $\operatorname{hExt}_{\mathcal{P}}^{*}\left(I^{(2)}, \mathbf{S}_{(2,2)}\right.$ ) (we take the Schur-de Rham complex here). If our groups were trivial, then the whole first term of the first spectral sequence would be trivial (the triviality of all columns except the first and last one follows from $\operatorname{Ext}_{\mathcal{P}}^{*}\left(I^{(2))}, F \otimes G\right)=0$ for $|F|,|G|>0$; hence the triviality of the last column follows from the exactness of the Schur-Koszul complex). Therefore the second spectral sequence would converge to 0. But according to [C2, Fact 8.1], the second term of this sequence has four non-trivial columns in which we have the groups $\operatorname{Ext}_{\mathcal{P}}^{*}\left(I^{(2)}, \Lambda^{2(1)}\right)$, hence it cannot converge to 0 . This contradiction finishes the proof.

\section{References}

[ABW] K. Akin, D.A. Buchsbaum, and J. Weyman, Schur functors and Schur complexes, Advances in Math. 44 (1982), no. 3, 207-278.

[Bo] G. Boffi, Universal filtration of Schur complexes, Proceedings of the AMS 119 (1993), no. 2, 351-355.

[CE] H. Cartan and S. Eilenberg, Homological algebra, Princeton Univ. Press, Princeton, NJ, 1956.

[C1] M. Chałupnik, Extensions of strict polynomial functors, Ann. Sci. École Norm. Sup. (4) 38 (2005), no. 5, 773-792.

[C2] M. Chałupnik, Schur-De-Rham complex and its cohomology, J. Algebra 282 (2004), no. 2, 699-727.

[C3] M. Chałupnik, Koszul duality and extensions of exponential functors, Advances in Math. 218 (2008), no. 3, 969-982. 
[Do] S. Donkin, On Schur algebras and related algebras IV: The blocks of the Schur algebras, J. Algebra 168 (1994) no. 2, 400-429.

[FFSS] V. Franjou, E.M. Friedlander, A. Scorichenko, and A. Suslin, General linear and functor cohomology over finite fields, Annals of Math. 150 (1999), no. 2, 663-728.

[FLS] V. Franjou, J. Lannes, and L. Schwartz, Autour de la cohomologie de MacLane des corps finis, Inventiones Math. 115 (1994), no. 3, 513-538.

[FS] E. Friedlander and A. Suslin, Cohomology of finite group schemes over a field, Inventiones Math. 127 (1997), no. 2, 209-270.

[GM] I. Gelfand and Y. Manin, Methods of homological algebra, Second edition, Springer Monogr. Math., Springer-Verlag, New York, 2003.

[JK] G. James and A. Kerber, The representation theory of the symmetric group, Encyclopedia of Mathematics and its Applications 16, Addison-Wesley Publ. Co., Reading, Mass., 1981.

[Ne] A. Neeman, Triangulated categories, Ann. of Math. Studies 148, Princeton Univ. Press, Princeton, NJ, 2001.

Marcin Chałupnik mchal@mimuw.edu.pl

Instytut Matematyki, University of Warsaw, ul. Banacha 2, 02-097 Warsaw, Poland 